\title{
Uma revisão dos fundamentos do estudo de inclusões fluidas aquosas e de petróleo
}

A REVIEW ON THE FUNDAMENTALS OF AQUEOUS AND PETROLEUM FLUID INCLUSION STUdY

André Luiz Silva Pestillho ${ }^{1}$, Lena Virgínia Soares Monteiro ${ }^{2}$

1 - Univ. São Paulo, Inst. Geociências. São Paulo-SP. Email: andrepestilho@usp.br

2 - Univ. São Paulo, Inst. Geociências. São Paulo-SP. Email: lena.monteiro@usp.br

ABSTRACT: This work seeks to introduce the conceptual basis and the philosophy behind the study of fluid inclusions. A brief look into the historical development and the current state of knowledge of the study of fluid inclusions are presented. From that point on, we address the concepts regarding the formation of fluid inclusions and the basic assumptions regarding the meaning of different fluid inclusion characteristics (Roedder's Rules). Finally, we present the basis for interpreting microthermometric data with examples from chemical systems from aqueous fluid inclusions, and a further review of the concepts involving the study of petroleum fluid inclusions.

Manuscrito:

Recebido: 01/08/2015

Corrigido: 11/11/2016

Aceito: 09/01/2017

Citation: Pestillo A.L.S., Monteiro L.V.S. 2017. Uma revisão dos fundamentos do estudo de inclusões fluidas aquosas e de petróleo. Terræ Didatica, 13(2):7192. <http://www.ige.unicamp.br/terraedidatica/> .

Keywords: Geology, fluid inclusions, economic geology, petroleum geology.

\section{Introdução}

O estudo de inclusões fluidas compreende um vasto campo das ciências geológicas, cujo desenvolvimento moderno iniciou-se há quase 200 anos (Roedder 1984, Bakker 1999). Os primeiros estudos analíticos, ainda no século XIX, limitavam-se a ensaios qualitativos, mas já permitiam o reconhecimento da formação de inclusões fluidas sob condições de pressão e temperatura maiores que as da superfície (Davy 1822) e a existência de diferentes tipos de fluidos aprisionados nos minerais (Brewster 1823).

O estudo petrográfico e microtermométrico de inclusões fluidas, como será abordado neste trabalho, utilizando uma interpretação numérica dos parâmetros físico-químicos, começou com o trabalho pioneiro de Sorby (Sorby 1858; Fig. 1). Contudo, somente a partir da segunda metade do século XX foi iniciado o uso sistemático de experimentos microtermométricos, em especial os de congelamento, para a caracterização da composição das inclusões fluidas (Roedder 1984). Ao mesmo tempo ocorreu grande avanço no desenvolvimento de novos métodos de análise química direta de inclusões fluidas (Roedder 1984).

A atual sistemática de estudo das inclusões fluidas compreende um vasto conjunto de métodos analíticos qualitativos e semiquantitativos, utilizando ensaios destrutivos e não destrutivos. Exemplos dos métodos qualitativos incluem a petrografia convencional (e.g., Kerkhof \& Hein 2001, Goldstein 2003), o uso de microscopia eletrônica por varredura (e.g., Pestilho 2008) e por transmissão (e.g., Viti \& Frezzotti 2001), o uso de catodoluminescência (e.g., Boiron et al. 1992) e o uso de petrografia quantitativa automatizada (e.g., Liu et al. 2004).
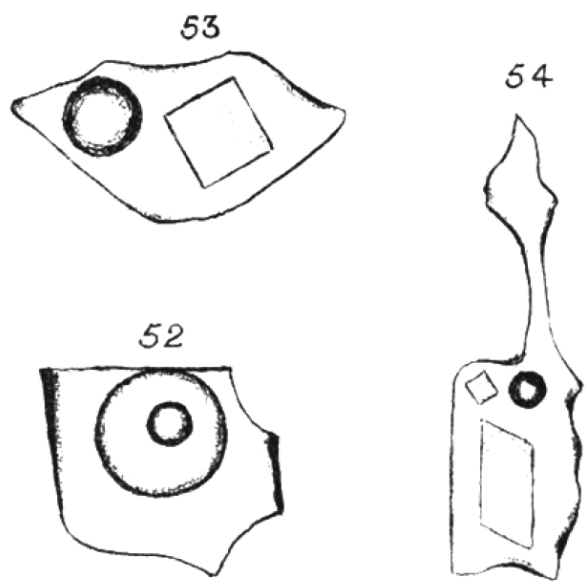

Figura 1. Ilustrações e descrições de inclusões fluidas por Henry Clifton Sorby: '52' (x2000) - "A fluid-cavity containing two fluids, in the quartz of porphyry at Cove, near Aberdeen"; '53' (x1600) e '54' (x800) - "Fluid-cavities containing various crystals, in quartz replacing feldspar, at Trevalgan, near St. Ives, Cornwall" (Sorby 1858). 
No campo das análises quantitativas e semiquantitativas existem métodos não destrutivos e destrutivos (Roedder 1984, Samson et al. 2003). Dentre os métodos não destrutivos, incluem-se exemplos como os da microtermometria (e.g., Roedder 1962, Roedder 1963a), das análises espectrofotométricas, que permitem caracterizar a composição de inclusões de óleo via espectrofotometria de fluorescência (e.g., Stasiuk \& Snowdon 1997), a análise indireta de voláteis aprisionados em inclusões fluidas aquosas por microespectrometria a laser RAMAN (e.g., Frezzoti et al. 2012) e análise microespectrométrica de inclusões individuais para a estimativa de composição de metais dissolvidos em inclusões aquosas (e.g., Kurosawa et al. 2003).

Exemplos de métodos destrutivos incluem a análise química total (bulk analysis) da composição eletrolítica (e.g., estimativa da composição de $\mathrm{Br}^{-}$, $\mathrm{Cl}^{-}$e I-) e de gases (e.g., estimativa da composição de $\mathrm{CH}_{4}, \mathrm{CO}_{2}$ e $\mathrm{H}_{2} \mathrm{~S}$ ) de inclusões aquosas através de técnicas cromatográficas (e.g., Roedder 1958, Roedder 1963b, Gleeson 2003, Salvi \& Williams-Jones 2003), a análise química total da composição molecular e de voláteis de inclusões de óleo a partir da cromatografia acoplada a espectrometria de massas (e.g., Murray 1957, Karlsen et al. 1993, Pang et al. 1998, Jones \& Macleod 2000, George et al. 2007, Jorge et al. 2011). Além disso, a análise química da composição de inclusões fluidas isoladas utilizando ablação a laser vem sendo cada vez mais utilizada, tanto na técnica associada à cromatografia em fase gasosa acoplada a espectrometria de massas para análise das inclusões fluidas de óleo (e.g., Volk 2010) como a técnica de plasma acoplado por indução a espectrometria de massas, utilizada para análise das inclusões fluidas aquosas (e.g., Gagnon et al. 2003).

Contudo, o aprofundamento dos métodos analíticos e de alguns tipos específicos de inclusões fluidas, como as de fundido silicático (silicate melt inclusions; Roedder 1984), foge à proposta deste trabalho. Revisões para aqueles leitores que desejem se aprofundar no assunto podem ser encontradas em Roedder (1984), Roedder \& Bodnar (1997) e Samson et al. (2003), muito embora estes sumários já se encontrem em grande parte desatualizados.

Este trabalho visa introduzir as bases conceituais e a filosofia de estudo de inclusões fluidas aquosas e de petróleo (óleo e gás) para estudantes de graduação e pós-graduação. Muitas vezes considerado um conhecimento avançado, que não é ensinado nos cursos de graduação e raramente está presente nos cursos de pós-graduação brasileiros, tal conhecimento é essencial ao estudo petrográfico e microtermométrico, e por sua vez é base de estudo de outros campos das ciências geológicas, como a petrologia, a geologia econômica e a geologia do petróleo. Esperamos com essa revisão tornar mais acessível o estudo de inclusões fluidas a todos os leitores de língua portuguesa.

\section{Conceitos da Interpretação de Inclusões Fluidas}

Com exceção dos minerais metamórficos, a maioria dos minerais cristaliza-se a partir de um fluido, seja ele um fluido aquoso ou um fundido silicático (Roedder 1984). Após o processo de cristalização, os minerais em praticamente todos os ambientes geológicos são fraturados, uma ou múltiplas vezes, permitindo a passagem de fluidos e a recristalização de microfraturas (Roedder 1984). Em ambos os casos, inclusões fluidas podem ser formadas pela preservação de gotículas de um fluido (líquido ou gasoso) em defeitos cristalinos (Fig. 2). Assim, o mineral que abriga a inclusão fluida é denominado mineral hospedeiro (Roedder 1984). Um terceiro caso ocorre quando o fraturamento e a formação das inclusões fluidas ocorrem simultâneos à cristalização de um mineral hospedeiro (Roedder 1984), como foi demonstrado por Roedder (1965).

A formação das inclusões fluidas em profundidade ocorre geralmente a partir do aprisionamento de um fluido homogêneo aquecido, a exemplo dos fluidos hidrotermais (Roedder \& Bodnar 1997) e diagenéticos (Goldstein \& Reynolds 1994). Depois de formadas, as inclusões tendem a permanecer como sistemas fechados, que permitem apenas a troca de energia na forma de calor (Roedder \& Skinner 1968, Roedder 1984).

Quando trazidas à superfície, seja por processos naturais ou por amostragem, o fluido aprisionado passa por mudança de fase (Roedder 1984). Esse processo decorre da mudança de estado termodinâmico do fluido de maiores para menores temperatura e pressão, em volume molar constante (i.e., processo isocórico). Tal mudança pode ser caracterizada pela dissociação de fases da matéria, como a formação de uma bolha (i.e., surgimento da fase vapor, como no exemplo da Fig. 3) ou, no caso de uma solução eletrolítica, pela precipitação de um ou mais sais, em sua maioria haletos deno- 


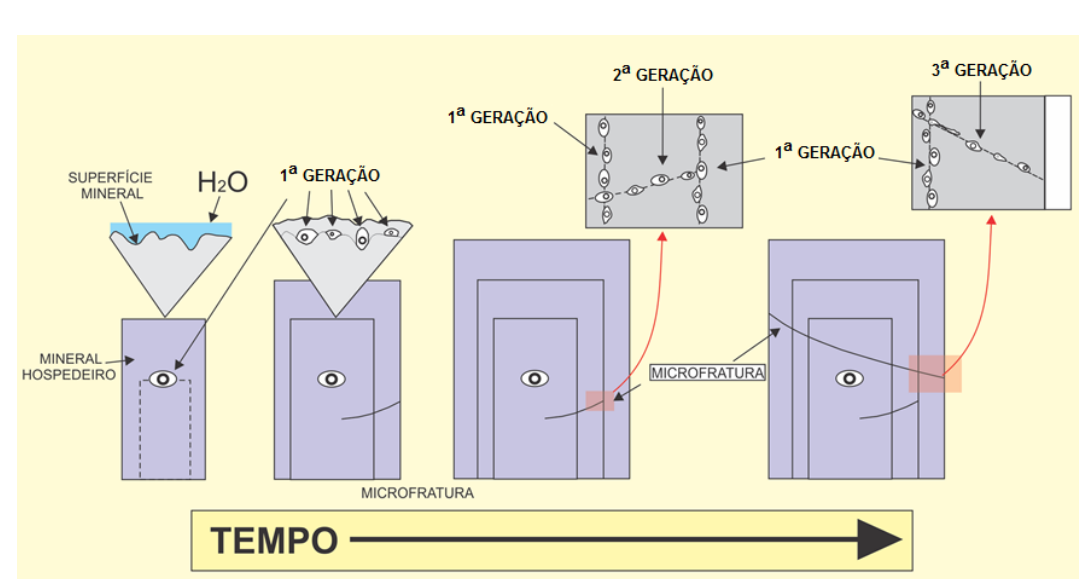

Figura 2. Formação de inclusões fluidas de acordo com a história de crescimento mineral. Durante o crescimento mineral, as inclusões fluidas podem se formar em defeitos microcristalinos nas zonas de crescimento ( $1^{\text {a }}$ geração), em microfratura formada concomitante ao crescimento mineral ( $2^{a}$ geração) ou mesmo pela recristalização de microfraturas originadas após o crescimento mineral ( $3^{a}$ geração; modificado de Bodnar 2003a).

minados minerais filhos, do inglês daughter minerals, associados à fase líquida. Em ambos os casos, essa mudança de estado deve-se à descompressão termal isocórica (Roedder 1984, Roedder \& Bodnar 1997).

A preservação das inclusões fluidas representa o

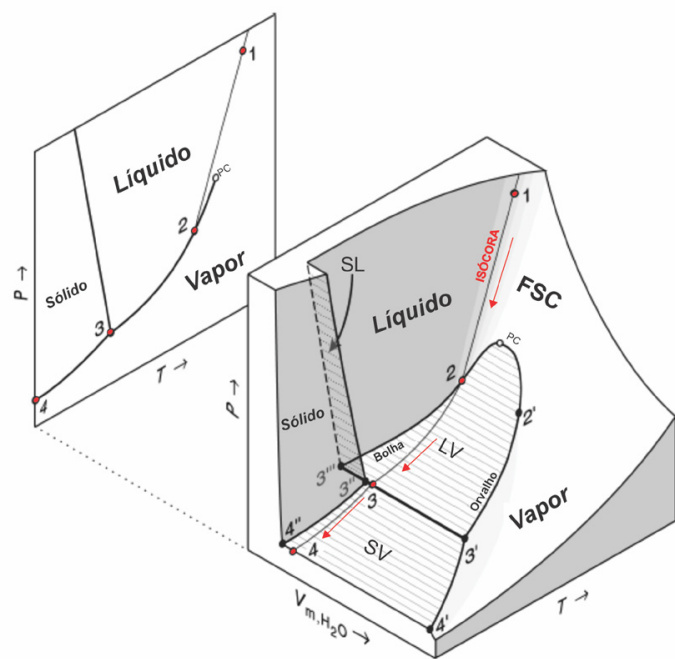

Figura 3. Diagrama de temperatura versus volume molar versus pressão, que mostra os limites das fases existentes vapor, líquido e sólido, além dos campos de coexistência de fases para o sistema $\mathrm{H}_{2} \mathrm{O}$ puro. Os pontos de 1 a 4 ilustram o processo de descompressão isocórica de uma inclusão fluida aprisionada em profundidade e, posteriormente, trazida às condições de superfície, como indicado pelas setas vermelhas: (1) condições iniciais de aprisionamento da inclusão fluida; (2) redução de temperatura acompanhada pela redução de pressão interna, que alcança condições para o surgimento da fase vapor; (3) redução contínua da temperatura leva ao alcance de condições para o aparecimento da fase sólida (i.e., gelo) no ponto triplo; (4) condições de coexistência da fase sólida e vapor (Diamond 2003a). registro de um fluido homogêneo aprisionado em um sistema fechado e de volume constante ao longo do tempo geológico e constitui a base interpretativa dos estudos de inclusões fluidas (Roedder 1984, Roedder \& Bodnar 1997, Goldstein \& Reynolds 1994, Bodnar 2003a). Contudo, nem sempre a formação de inclusões fluidas ocorre a partir de um fluido homogêneo, e muitas vezes as inclusões fluidas não são preservadas (Roedder 1984, Goldstein \& Reynolds 1994, Roedder \& Bodnar 1997, Bodnar 2003a). Processos de imiscibilidade pré-aprisionamento e de modificação pós-aprisionamento do fluido são os dois principais fenômenos responsáveis pela interpretação errônea dos dados fornecidos pelas inclusões fluidas (Roedder 1984).

Por esse motivo, idealmente, as inclusões fluidas selecionadas como possíveis representantes do fluido original associado ao sistema de interação fluido-rocha durante um processo geológico (i.e., diagênese, hidrotermalismo, metamorfismo, etc.) deve seguir um conjunto de premissas sobre a natureza do fluido aprisionado e o modo de formação das inclusões fluidas, de modo que conclusões concisas acerca da história geológica do sistema possam ser elaboradas (Roedder \& Bodnar 1997). Esse conjunto de premissas, muitas vezes denominadas Regras de Roedder (Bodnar 2003a), em especial aquelas apontadas para depósitos minerais (Roedder \& Bodnar 1997) e processos diagenéticos (Goldstein \& Reynolds 1994), incluem:

(I) uma amostra representativa do fluido associado ao processo diagenético ou hidrotermal foi aprisionada durante o crescimento de cristais;

(II) nada foi adicionado ou removido da inclusão após o aprisionamento do fluido;

(III) o volume da cavidade que aprisiona o fluido não aumentou ou diminuiu após o aprisionamento;

(IV) as relações temporais e espaciais entre o evento de aprisionamento do fluido e os processos geológicos associados, como a 
deposição do minério ou o momento de preenchimento de um reservatório de petróleo, são conhecidas;

os efeitos da pressão na formação das inclusões são insignificantes ou conhecidos.

A premissa do aprisionamento de um fluido homogêneo (Premissa I) pode não ser verdadeira se de algum modo o fluido inicial separou-se em dois fluidos ou mais, devido ao processo de imiscibilidade pré-aprisionamento ou mesmo devido à natureza do aprisionamento (Roedder \& Bodnar 1997).

$\mathrm{O}$ aprisionamento heterogêneo devido ao efeito de adsorção de componentes a parede dos cristais (thin layer boundary) é similar à cromatografia por camadas delgadas (thin layer cromatography), embora reconhecido como um efeito que possivelmente é responsável pela diferenciação entre o fluido aquoso original (e.g., de um sistema hidrotermal) e o aprisionado nas inclusões fluidas (Roedder \& Bodnar 1997). Contudo, nenhuma evidência sugere que tal fenômeno afete consideravelmente as inclusões fluidas aquosas (Roedder \& Bodnar 1997).

A forma mais comum de aprisionamento heterogêneo decorre da imiscibilidade de fluidos seja pela descompressão (e.g., $\mathrm{H}_{2} \mathrm{O}$ líquido e vapor de água, etc.; Fig. 4A), pela hipersaturação em sais que causam a precipitação de sólidos acidentais (Fig. 4B) ou pelo aprisionamento de fluidos de composição distinta (e.g., $\mathrm{H}_{2} \mathrm{O}$ e $\mathrm{CO}_{2}, \mathrm{H}_{2} \mathrm{O}$ e óleo, metano e $\mathrm{H}_{2} \mathrm{O}$ líquido, óleo e metano, etc.; Roedder 1984). Em alguns casos, os fluidos heterogêneos hipersaturados em sais podem acarretar no aprisionamento de cristais que ocupam quase todo o volume da cavidade da inclusão fluida (Schiffries 1990).

Contudo, em alguns casos, o fenômeno de aprisionamento heterogêneo pode fornecer informação sobre processos de imiscibilidade pré-aprisionamento (i.e., nos casos de imiscibilidade líquidovapor de fluidos de mesma composição química; Roedder \& Bodnar 1980, Roedder 1984, Goldstein $\&$ Reynolds 1994). Isso ocorre especialmente quando se tratam de fluidos de diferentes composições, o que não contraria a Premissa $I$, pois tratam-se $a$ priori de dois fluidos homogêneos migrando numa mistura heterogênea, como água e óleo ou água e $\mathrm{CO}_{2}$ (Roedder \& Bodnar 1980, Roedder 1984, Goldstein \& Reynolds 1994, Diamond 2003b). Mais ainda, aprisionamento de fluidos imiscíveis pode permitir o estabelecimento das pressões e temperaturas de aprisionamento (Bodnar \& Roe-
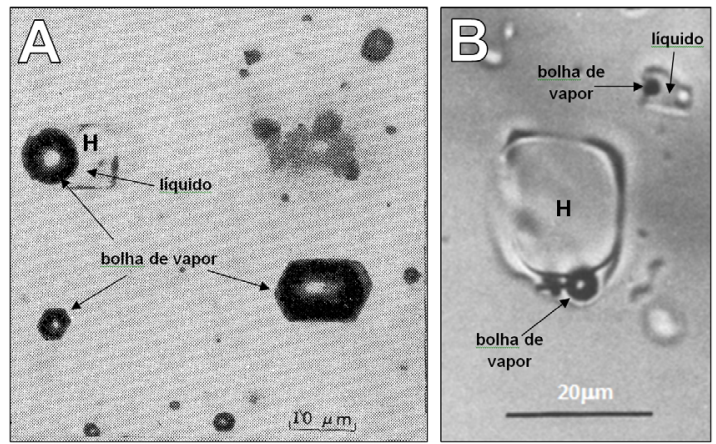

Figura 4. Exemplos de inclusões fluidas formadas posteriormente ao processo de imiscibilidade de um fluido homogêneo: (A) inclusões fluidas com densidades variadas, possivelmente formadas pela ebulição (boiling) de um fluido aquoso homogêneo (Roedder 1971); (B) inclusões fluidas com desproporção de minerais filhos aprisionados, que foram formadas devido à precipitação dos sais (Balhaus \& Stumpfl 1986). Abreviação: H - halita.

dder 1980, Roedder 1984), como por exemplo, no caso de óleo e água (Munz, 2001).

Ainda no que diz respeito à Premissa I é importante notar o caso das inclusões fluidas advindas da recristalização de pseudomorfos (Goldstein 2001). Em particular nos ambientes sedimentares carbonáticos, inclusões fluidas que se formam junto ao crescimento de grãos diagenéticos podem ser substituídas junto ao grão, que se torna um pseudomorfo, pelo processo de recristalização (i.e., na diagênese dos carbonatos corresponde à substituição de minerais de mesma composição), aprisionando um novo fluido homogêneo associado às novas condições diagenéticas (Goldstein 2001). Isso parece contrariar a Premissa I, contudo as inclusões geradas foram aprisionadas junto ao mineral substituto, não inviabilizando assim essa premissa (e.g., Goldstein 2001).

A premissa de que as inclusões fluidas constituem um sistema fechado (Premissa II) pode não ser atendida em alguns casos. Quando uma inclusão fluida é submetida a condições de temperatura e pressão nas quais ocorre o seu vazamento (leakage), o fenômeno mais comum é a decriptação, onde todo o fluido aprisionado vaza (Roedder 1984, Bodnar 2003b).

Já foi demonstrado que as inclusões geralmente não vazam água mesmo quando submetidas a condições de temperatura e pressão maiores do que as do seu aprisionamento (Roedder \& Skinner 1968). No entanto, em condições de elevada temperatura e pressão (e.g., inclusões de depósitos minerais metamorfisados na fácies anfibolito médio 
a superior) pode haver remobilização de água (ou hidrogênio) tanto para dentro como para fora das inclusões fluidas (Hall \& Bodnar 1990, Hall et al. 1991, Mavrogenes \& Bodnar 1994, Hall \& Sterner 1995). Alguns experimentos corroboram essa interpretação, onde foi reportado, por exemplo, o fenômeno de difusão em temperaturas da ordem de $600{ }^{\circ} \mathrm{C}$ (Doppler et al. 2013) e $625^{\circ} \mathrm{C}$ (Vitik \& Bodnar 2000).

A premissa da preservação do volume constante (Premissa III) também pode não ser alcançada devido às modificações pós-aprisionamento. Entre os dois principais processos responsáveis pela modificação mecânica das inclusões fluidas está o reequilíbrio das inclusões pelos processos de estiramento (streching) e o estrangulamento (necking down; Roedder 1984, Goldstein \& Reynolds 1994, Bodnar 2003c).

O processo de estrangulamento de inclusões fluidas ocorre geralmente em inclusões alongadas, que são divididas naturalmente pelo processo de recristalização do quartzo (Roedder 1984, Bodnar 2003c). Isso ocorre para poder ajustar as inclusões a uma menor tensão superficial (e.g., como a forma que uma gota de óleo toma quando envolta pela água obtendo uma forma esférica; Roedder 1984, Goldstein \& Reynolds 1994, Bodnar 2003c). Esse processo também ocorre durante o aprisionamento primário de fluidos em microfraturas, mas neste caso são seladas diferentes frações de um fluido homogêneo e, como resultado, as inclusões formadas são representativas do fluido original (Roedder 1984, Goldstein \& Reynolds 1994, Bodnar 2003c). Contudo, se esse processo ocorre após a nucleação de uma fase (i.e., nucleação de bolha ou precipitação de um sal), as inclusões resultantes terão composições diferentes e irão apresentar resultados microtermométricos errôneos (Roedder 1984, Goldstein \& Reynolds 1994, Bodnar 2003c). No caso da precipitação do sal posteriormente ao aprisionamento, nem mesmo a quantificação da salinidade das inclusões resultantes dos estrangulamentos pode ser aproveitada (Bodnar 2003c).

$\mathrm{O}$ processo de estiramento corresponde à expansão volumétrica da cavidade hospedeira do fluido (Roedder 1984, Goldstein \& Reynolds 1994, Bodnar 2003c). Essa expansão, que é causada pelo aquecimento e reajuste da tensão superficial às novas condições de temperatura, provoca a expansão do fluido aprisionado (Roedder 1984, Rodder \& Bodnar 1997, Bodnar 2003b). Como resultado, as informações termométricas ficam comprome- tidas, pois com o aumento do volume molar há o aumento proporcional da temperatura, como foi postulado inicialmente pela lei dos gases ideais e pela relação da equação de estado de Johannes Diderik van der Waals (Maxwell 1874), assim a temperatura refletida pelas inclusões reequilibradas é maior que a original (Bodnar 2003c).

Contudo, já foi demonstrado que o grau de modificação das inclusões, em especial devido ao processo de estiramento, deriva principalmente do aumento da plasticidade e viscosidade do mineral (Tugarinov \& Naumov 1970, Faiziev \& Alidovoc 1976; Fig. 5). Por esse motivo, inclusões fluidas formadas em sulfatos e calcita, típicos cimentos em ambientes sedimentares, tem uma maior susceptibilidade ao reequilíbrio por estiramento do que aquelas aprisionadas em quartzo (Tugarinov \& Naumov 1970, Prezbindowski \& Larese 1987).

Além disso, o tamanho das inclusões, de fato, favorece o reequilíbrio (Fig. 6) sendo que as inclusões com os maiores volumes molares são as mais afetadas pelos processos de estiramento (Prezbindowski \& Larese 1987) e decrepitação (Bodnar et al. 1989).

A forma das inclusões fluidas também é um parâmetro importante a ser considerado na preservação das inclusões pós-aprisionamento (Bodnar et al. 1989). A relação entre a forma das inclusões

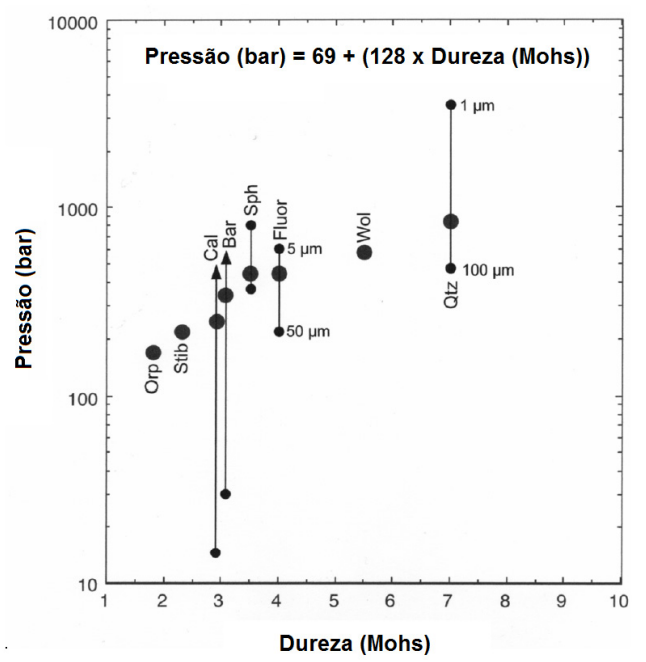

FIGURA 5. Relação entre a pressão interna necessária para o reequilíbrio de uma inclusão fluida e a dureza de Mohs, para diferentes tamanhos de inclusões fluidas (Tugarinov \& Naumov 1970, Bodnar 2003c). Dados para quartzo são de Bodnar et al. (1989); dados de fluorita e esfalerita são de Bodnar \& Bethke (1984); de barita são de Ulrich \& Bodnar (1988); e de calcita foram estimados a partir de Hall et al. (1993) e Prezbindowski \& Larese (1987). 


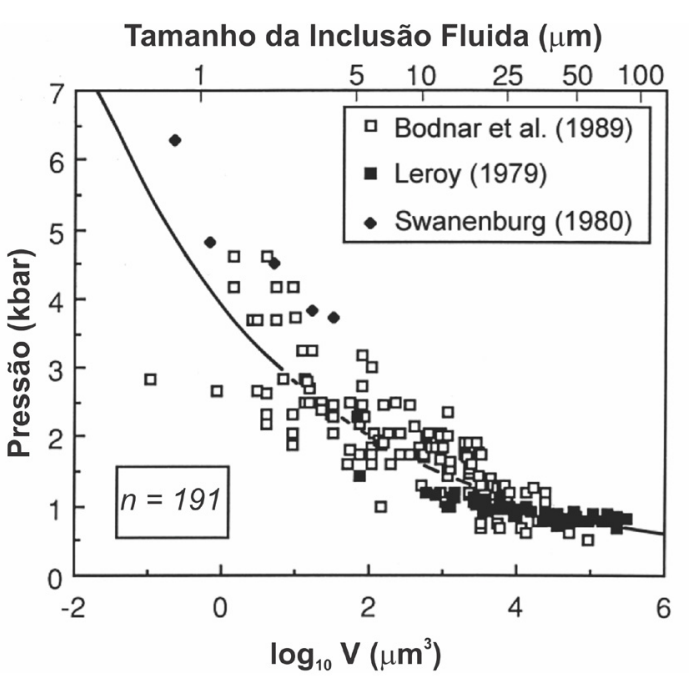

Figura 6. Diagrama do volume molar versus pressão indicando a susceptibilidade à deformação das inclusões com o tamanho (Bodnar et al. 1989).

fluidas e a quantidade de pressão necessária para iniciar o reequilíbrio geralmente é consistente com modelos teóricos de deformação que indicam que a tensão fica concentrada nos cantos acentuados e que a tensão atinge valores infinitesimais nas pontas de inclusões com formato estrelado, também denominado tentacular, ou nos cantos de inclusões muito angulares (Prezbindowski \& Tapp 1991).

A quarta premissa (Premissa IV), no entanto, diz respeito ao estabelecimento da relação entre os paleofluidos e a evolução paragenética de um depósito mineral hidrotermal ou da cimentação de um reservatório (Roedder 1984, Roedder \& Bodnar 1997, Wilkinson 2001, Goldstein \& Reynolds 1994, Goldstein 2001). A caracterização da ordem de eventos relativos aos estágios de alteração hidrotermal ou diagenéticos até a deposição do minério ou o preenchimento de um reservatório deve ser realizada previamente ao estudo de inclusões fluidas (Burruss 1981, Roedder \& Bodnar 1997, Wilkinson 2001, Munz 2001).

No caso de um depósito mineral metálico, um determinado conjunto de inclusões que foi aprisionado durante a cristalização do mineral de ganga associado ao minério (e.g., quartzo em veios hospedeiros de ouro) pode não fornecer informações diretamente relacionadas à precipitação dos metais (i.e., condições de pressão e composição do fluido durante a mineralização; Wilkinson 2001). Muitas vezes o minério está superposto à alteração hidrotermal disseminada e fissural, na qual formaram-se os minerais que hospedam as inclusões fluidas viáveis ao estudo (Wilkinson 2001). Uma solução prática é o estudo direto de inclusões hospedadas nos próprios minerais de minério (e.g., em esfalerita - $(\mathrm{Zn}, \mathrm{Fe}) \mathrm{S}$, pirargirita - $\mathrm{Ag}_{3} \mathrm{SbS}_{3}$, wolframita - $(\mathrm{Fe}, \mathrm{Mn}) \mathrm{WO}_{4}$, cinábrio - $\mathrm{HgS}$, estibinita $-\mathrm{Sb}_{2} \mathrm{~S}_{3}$, pirita $-\mathrm{FeS}_{2}$, hematita $-\mathrm{Fe}_{2} \mathrm{O}_{3}$, etc.), o que pode ser feito com uso de microscopia óptica convencional, no caso de minerais translúcidos como a esfalerita, ou a partir da microscopia com luz infra-vermelha transmitida (e.g., Campbel et al. 1984).

No caso de bacias sedimentares, esse problema é contornado com o estudo de inclusões fluidas de óleo e as inclusões aquosas co-genéticas, que podem representar o registro direto de um sistema petrolífero quando ativo (Munz 2001), especialmente quando associadas a uma paleocoluna de óleo (e.g., George et al. 2004).

Quanto à última premissa (Premissa V), como as inclusões fluidas geralmente não fornecem uma informação direta da pressão de aprisionamento dos fluidos, muitos trabalhos tendem a abandonar o uso da geobarometria de inclusões fluidas (Golstein \& Reynolds 1994). Muito embora em alguns sistemas hidrotermais os gradientes de pressão não sejam, em geral, relevantes como mecanismos de precipitação do minério, em outros sistemas hidrotermais específicos, como no sistema magmático-hidrotermal, a importância da estimativa da profundidade de expulsão dos fluidos hidrotermais foi demonstrada (Roedder \& Bodnar 1997). A pressão de aprisionamento, no entanto, pode ser determinada quando há pares de fluidos de composições distintas (e.g., $\mathrm{H}_{2} \mathrm{O}$ e $\mathrm{CO}_{2}, \mathrm{H}_{2} \mathrm{O}$ e $\mathrm{CH}_{4}, \mathrm{H}_{2} \mathrm{O}$ e óleo) imiscíveis aprisionados separadamente no mesmo mineral hospedeiro, pois o conjunto de seu comportamento isovolumétrico molar (i.e., isocórico) permite estimar a pressão e temperatura de aprisionamento (Roedder \& Bodnar 1980).

Por fim, é importante ter em mente que essas premissas são guias ao estudo das inclusões fluidas, pois a maioria das inclusões fluidas não cumpre uma ou mais das três primeiras premissas. Contudo, se os desvios forem conhecidos e, o mais importante, se estes desvios podem ser estimados, os resultados podem ainda fornecer informações úteis ao processo interpretativo (Roedder \& Bodnar 1997).

\section{Relações de Mudanças de Fases em Inclusões Fluidas}

Uma vez que o estudo petrográfico tenha sido realizado a contento, dada às condições adequadas (e.g., abundância de inclusões fluidas da amostra, tamanho das inclusões fluidas da amostra, etc.) o 
estudo de inclusões fluidas pode prosseguir com o emprego de métodos analíticos quantitativos e semiquantitativos (Roedder \& Bodnar 1997). Usualmente o estudo petrográfico precede à análise microtermométrica, dado à grande quantidade de informações que este método fornece, como será visto adiante, além do fato de ser um ensaio não destrutivo e barato (Roedder 1984).

Com base nos princípios de Roedder, o protocolo do estudo microtermométrico consiste em medir a temperatura na qual ocorrem os pontos de mudanças de fases devido ao aquecimento ou ao resfriamento do fluido aprisionado na inclusão fluida (Roedder 1984).

Informações importantes a respeito do fluido podem ser estimadas, dadas às condições ideais, tais como (1) pressão e temperatura de aprisionamento da inclusão fluida, (2) a composição em termos do sistema químico dominante e da salinidade, e (3) densidade do fluido (Roedder 1984, Goldstein \& Reynolds 1994). Estas informações, quando associadas a uma geração específica de inclusões fluidas, permitem fazer inferências sobre a origem e a modificação de um fluido durante a evolução de um sistema de interação fluido-rocha (Goldstein \& Reynolds 1994, Roedder \& Bodnar 1997).

Para tais estimativas, os dados de temperatura são interpretados com base em equações de estado e diagramas de fase que permitam a reconstrução do campo de Pressão-Volume-Temperatura-Composição que rege o comportamento do fluido (Goldstein \& Reynolds 1994). Tal detalhamento pode ser encontrado na literatura, como os exemplos de: inclusões de água pura (e.g., Diamond 2003a); inclusões fluidas aquosas compostas por solução eletrolítica (e.g., Bodnar 2003b); inclusões fluidas portadoras de gases, tais como $\mathrm{CO}_{2}$ e $\mathrm{CH}_{4}$ (e.g., Diamond 2003b); inclusões fluidas portadores de óleo (e.g., Munz 2001, Burruss 2003).

Várias medidas da temperatura nas quais ocorrem as mudanças de fase podem ser obtidas por meio de observações ao microscópio petrográfico, a fim de descrever a topologia de um sistema químico, com os respectivos campos de estabilidade das fases formadas, a partir da mudança de estado de um fluido aprisionado como uma fase homogênea (Diamond 2003a). Como exemplo, podem ser observadas: (i) a temperatura de homogeneização $\left(\mathrm{Th}^{1}\right)$ de inclusões fluidas aquosas e de óleo;

(ii) a temperatura de fusão inicial (Tfi), medida exclusivamente a partir de observações de inclusões fluidas aquosas, que pode ser interpretada como a temperatura do eutético (Te) em inclusões fluidas que não portam uma fase gasosa (e.g., $\left.\mathrm{CH}_{4}\right)$;

(iii) a temperatura de fusão final de um sólido ( $\mathrm{Tf}$ ) medida em inclusões fluidas aquosas, seja o sólido gelo (Tfg), sais hidratados (e.g., hidrohalita, $\mathrm{NaCl} \cdot 2 \mathrm{H}_{2} \mathrm{O}$ - Tfhh) ou hidrato de gás, no caso de inclusões portadoras de gases (e.g., clatratos de $\mathrm{CH}_{4}$ e/ou $\mathrm{CO}_{2}$, designados como Tfcla);

(iv) outro parâmetro microtermométrico raramente utilizado no estudo de inclusões fluidas aquosas é a temperatura de nucleação (Tn), que corresponde à temperatura em que se formam as fases durante o resfriamento de uma inclusão fluida (e.g., $\left.\mathrm{Tn}_{\text {gelo }}\right)$.

Alguns autores ainda fazem o uso da medição da temperatura de decriptação da inclusão fluida (Td), que pode ser medida tanto em inclusões fluidas aquosas como de óleo para complemento da análise microtermométrica. A Td corresponde à temperatura na qual a inclusão fluida se rompe durante o aquecimento, o que pode ocorrer antes que a temperatura de homogeneização seja atingida, ou mesmo no uso prático de estimativa rápida da temperatura de homogeneização de fluidos utilizando decriptômetros acústicos (Roedder 1984).

\subsection{Temperaturas de Homogeneização}

A temperatura de homogeneização ou temperatura de saturação é definida como a temperatura na qual um fluido composto por diferentes fases torna-se homogêneo (Roedder \& Bodnar 1980, Roedder 1984, Goldstein \& Reynolds 1994). Em teoria, o fenômeno da homogeneização obtido pela análise microtermométrica representa uma regressão à condição de temperatura do momento no qual a inclusão fluida foi formada (Roedder \& Bodnar 1980, Roedder 1984, Goldstein \& Reynolds 1994). Contudo, como mencionado, a temperatura 1 Adaptação para o português das abreviações, quantidades e siglas propostas
por Diamond (2003c). 
de homogeneização não reflete a temperatura de aprisionamento (Roedder \& Bodnar 1980, Roedder 1984, Goldstein \& Reynolds, 1994).

Para fins ilustrativos tomaremos aqui como base um sistema simples, composto de $\mathrm{H}_{2} \mathrm{O}$ pura (Fig. 7; Diamond 2003a). O envelope que limita os estados das fases aprisionadas (i.e., líquido, sólido, vapor e o locus do fluido supercrítico) é denominado isopleta, que pode ser também um ponto ou linha desde que seja específico para uma mesma composição (Diamond 2003a). As inclusões fluidas, por apresentarem volume molar constante depois de formadas, seguem uma trajetória topológica linear no diagrama P-T (Pressão e Temperatura) como resposta às mudanças dos parâmetros de pressão ou temperatura (Diamond 2003a).

Depois de formadas pelo aprisionamento de um fluido homogêneo, quando trazidas à superfície, a redução de temperatura leva a uma redução diretamente proporcional de pressão interna da inclusão de acordo com uma isócora do diagrama P-T (Diamond 2003a). Levando-se em conta uma inclusão fluida rica na fase líquida (i.e., com reduzido volume molar de $\mathrm{H}_{2} \mathrm{O}$, por exemplo, entre 18 a $24 \mathrm{~cm}^{3} \cdot \mathrm{mol}^{-1}$ ), com a redução progressiva da temperatura o fluido atinge condições P-T da curva L-V (i.e., dos líquidos e vapores) de modo que o fluido antes homogêneo nucleia uma bolha, que essencialmente consiste de um vácuo ou vapor de água formado pela compressão do líquido contra as paredes da cavidade (Diamond 2003a). Já inclusões

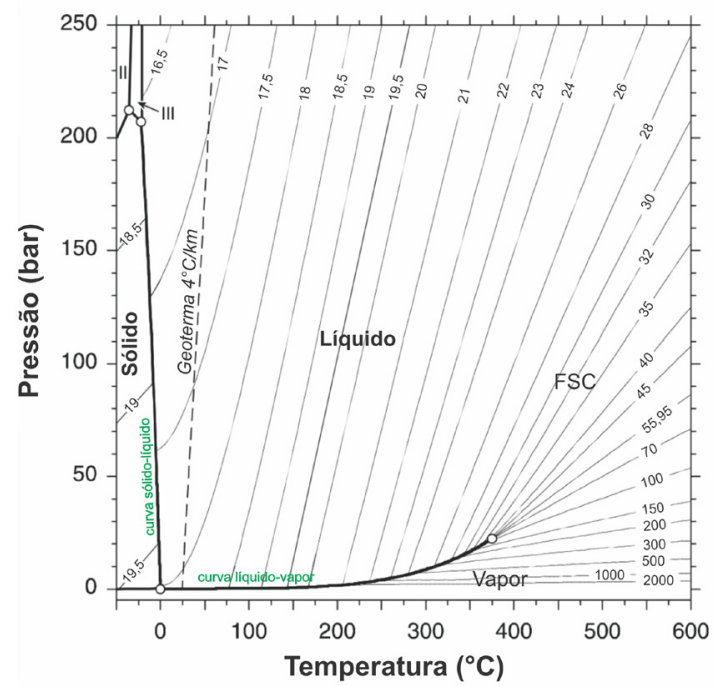

Figura 7. Diagrama de temperatura versus pressão mostrando os campos de estabilidade do estado sólido, líquido, vapor e o locus do fluido supercrítico (FSC) para o sistema $\mathrm{H}_{2} \mathrm{O}$ puro. As linhas de inclinação oblíqua são isócoras com valores de volume molar em kg. $\mathrm{mol}^{-1}$ (Diamond 2003a). com grande valor de volume molar de $\mathrm{H}_{2} \mathrm{O}$ (e.g. $500 \mathrm{a} 2000 \mathrm{~cm}^{3}$. $\mathrm{mol}^{-1}$ ) homogeneízam pela curva do ponto de bolha, pela condensação de um vapor (Diamond 2003a). Existem ainda algumas inclusões fluidas que homogeneízam para um fluido supercrítico dado seu volume molar e condição de aprisionamento (Roedder 1984, Goldstein \& Reynolds 1994, Diamond 2003a).

Devido à inclinação das isócoras (i.e., as trajetórias topológicas que uma determinada inclusão com um valor de densidade fixo segue quando ocorre a modificação de temperatura e pressão), a temperatura e pressão de aprisionamento das inclusões fluidas é quase sempre maior que o valor de Th (Roedder 1984, Goldstein \& Reynolds 1994 , Bodnar 2003b). Por isso considera-se o valor de Tht como o valor mínimo de temperatura de aprisionamento do fluido. Em inclusões fluidas cogenéticas com o mineral hospedeiro, Tht também é o valor de temperatura mínimo para a formação do mineral (Roedder 1984, Goldstein \& Reynolds 1994, Bodnar 2003b). Isso é ainda mais evidente no caso da homogeneização de inclusões fluidas de óleo que possuem isócoras muito inclinadas, sendo que seu valor de Th geralmente é menor (e.g., usualmente 10 a $20^{\circ} \mathrm{C}$ ) do que o estimado para inclusões fluidas aquosas cogenéticas (Munz 2001, Burruss 2003).

A exceção pode ocorrer em inclusões de água pura ou de baixa salinidade, formadas em baixa ou moderada temperatura $\left(<150{ }^{\circ} \mathrm{C}\right)$, quando a inclinação das isócoras é praticamente vertical, o que não há uma diferença significativa entre a temperatura de homogeneização e a real temperatura de aprisionamento das inclusões podendo ser consideradas aproximadamente iguais (Roedder 1984, Goldstein \& Reynolds 1994, Goldstein 2001, Bodnar 2003b).

No caso de inclusões com mais de uma fase, por exemplo, em uma inclusão constituída de sólido, líquido e vapor, é importante notar que a temperatura de homogeneização entre as fases ocorre de modo parcial em temperaturas diferentes, com a única exceção do ponto triplo (Bodnar 2003b, Diamond 2003a). Por esse motivo, são medidas também as temperaturas de homogeneização parcial entre a fase líquida e vapor (Th L-V) e a fase líquida e sólida (Th L-S). A temperatura da última fase a se homogeneizar, neste caso, corresponde à temperatura de homogeneização total (Tht) (Roedder 1984, Goldstein \& Reynolds 1994, Bodnar 2003b, Diamond 2003a). 
A obtenção da temperatura de aprisionamento (Tap), no entanto, depende de um prévio conhecimento da pressão de aprisionamento (Roedder \& Bodnar 1980). Uma alternativa viável para a obtenção de Tap é o uso de gradientes geotérmicos como parâmetro para a estimativa da pressão de aprisionamento, o que em bacias sedimentares pode ser feito com base na modelagem da curva de compactação e soterramento (Goldstein \& Reynolds 1994, Goldstein 2001).

Por fim, como já foi citado (item 2), no caso singular do aprisionamento contíguo de dois fluidos imiscíveis de natureza distinta, o cruzamento das isócoras (Fig. 8) de ambas as inclusões refletem a exata pressão e temperatura de aprisionamento (Roedder \& Bodnar 1980).

\subsection{Temperatura do Eutético do Sistema Químico de Inclusões Fluidas Aquosas}

A estimativa da composição química dos cátions maiores que compõem uma solução eletrolítica pode ser definida através da determinação da temperatura de eutético (Te; Roedder 1984, Goldstein \& Reynolds 1994). A temperatura do eutético corresponde à temperatura mínima de estabilidade líquida em um sistema químico. Um fluido de uma inclusão aquosa totalmente congelada quando aquecido irá fundir quando atingir o valor de Te (Roedder 1984, Goldstein \& Reynolds 1994).

Contudo, devido aos efeitos de metaestabilidade e a dificuldade de observação de Te, especialmente para as inclusões $<5 \mu \mathrm{m}$, muitas vezes é medido o valor de Tfi, que é correlacionado aos vários valores de Te que constam na literatura (Roedder 1984, Goldstein \& Reynolds 1994).

Muitas inclusões apresentam um comportamento metaestável devido ao fenômeno de histerese (Roedder 1984, Goldstein \& Reynolds 1994). A histerese sempre ocorre durante o congelamento das inclusões fluidas, razão pela qual a temperatura de nucleação das fases nunca equivale ao ponto de mudança de fases. Por esse motivo a tomada de temperatura das mudanças de fase é sempre feita durante o descongelamento (Roedder 1984, Goldstein \& Reynolds 1994).

Alguns procedimentos microtermométricos (e.g., uso de baixas taxas de aquecimento, uso de ciclos de aquecimento e resfriamento) podem reduzir a ocorrência desse fenômeno durante o aquecimento, mas não o evitar por completo (Roedder 1984, Goldstein \& Reynolds 1994). A consideração

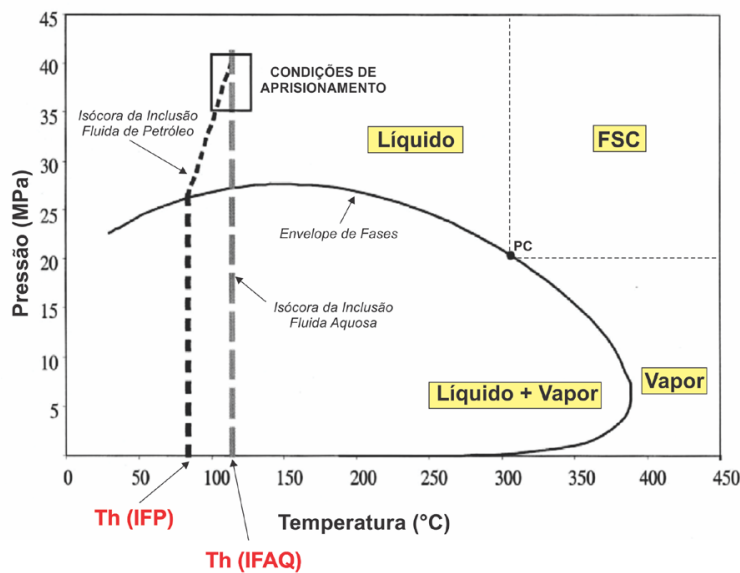

Figura 8. Diagrama de pressão versus temperatura, mostrando o campo de ocorrência das fases líquida, vapor e supercrítica (i.e., fluido supercrítico, FSC) para um petróleo do tipo black oil e o exemplo do cruzamento de isócoras de dois tipos de fluido: petróleo (inclusão fluida de petróleo, IFP) e água (inclusão fluida aquosa, IFAQ) (modificado de Munz 2001).

de temperaturas de mudança de fase ocorridas em condição de equilíbrio metaestável, especialmente em baixa temperatura, constitui um fenômeno normal e que quando compreendido pode ser utilizado na interpretação dos dados microtermométricos. Detalhamento e explicação do fenômeno podem ser encontrados em Roedder (1984).

Complicações adicionais advém de valores de temperatura de eutético muito próximos, a exemplo dos sistemas KCl-NaCl- $\mathrm{H}_{2} \mathrm{O}$, cujo eutético ocorre a $-22,9^{\circ} \mathrm{C}$, e NaCl-H2O, cujo eutético ocorre em $-21,2^{\circ} \mathrm{C}$, o que torna impossível sua distinção microtermométrica em inclusões fluidas de baixa salinidade, compostas apenas da fase líquida e vapor a temperatura ambiente (Goldstein \& Reynolds 1994, Bodnar 2003b). Nestes casos, a determinação da composição dos sistemas somente é possível quando há a presença de um ou mais sólidos filhos (silvita \pm halita) que possam ser identificados tanto pelo seu comportamento microtermométrico, como descrito em Bodnar (2003b), como por métodos alternativos, como o uso da microespectroscopia a laser Raman (e.g., Frezzotti et al. 2012).

O sistema $\mathrm{CaCl}_{2}-\mathrm{NaCl}-\mathrm{H}_{2} \mathrm{O}$ possui um ponto eutético estável em $-52{ }^{\circ} \mathrm{C}$, além de ser composto pelos subsistemas $\mathrm{CaCl}_{2}-\mathrm{H}_{2} \mathrm{O}$, cujo eutético é - 49,9 ${ }^{\circ} \mathrm{C}$, e o sistema $\mathrm{NaCl}-\mathrm{H}_{2} \mathrm{O}$ com eutético em $-21,2$ ${ }^{\circ} \mathrm{C}$ (Bodnar 2003b).

Em caso de inclusões fluidas portadores de misturas de gases (e.g., $\mathrm{CH}_{4}-\mathrm{CO}_{2}$ ), a temperatura de fusão inicial observada é sempre menor do que 
a de um sistema unário (e.g., $\mathrm{CO}_{2}$ puro) ou binário composto por um gás e água (e.g., $\mathrm{CO}_{2}-\mathrm{H}_{2} \mathrm{O}$, $\mathrm{CH}_{4}-\mathrm{H}_{2} \mathrm{O}$; Diamond 2003b). Essa é uma característica geral de todos os sistemas binários nas quais soluções sólidas não ocorrem (Diamond 2003b).

Ao contrário do ponto eutético de um sistema unário ou binário com um gás, o ponto eutético dos fluidos com mistura de gases é marcado pela fusão da fase sólida do gás de menor temperatura, o que no caso do sistema $\mathrm{CH}_{4}-\mathrm{CO}_{2}-\mathrm{H}_{2} \mathrm{O}$, ocorre ao redor da temperatura de ponto quádruplo do sistema $\left(-183^{\circ} \mathrm{C}\right)$, que não pode ser atingida devido à limitação do método microtermométrico convencional de congelamento das inclusões fluidas com nitrogênio líquido (Diamond 2003b). Assim, no sistema $\mathrm{CH}_{4}-\mathrm{CO}_{2}-\mathrm{H}_{2} \mathrm{O}$, a temperatura de fusão do $\mathrm{CO}_{2}$ sólido, que marca o eutético do sistema $\mathrm{CO}_{2}-\mathrm{H}_{2} \mathrm{O}$ e $\mathrm{CO}_{2}$-puro em $-56,6{ }^{\circ} \mathrm{C}$ pode ocorrer num intervalo de temperatura entre $-56,6^{\circ} \mathrm{C}$ e -85 ${ }^{\circ} \mathrm{C}$ para uma composição de $\mathrm{CH}_{4}$ que varia entre 0 e $82 \%$ (Diamond 2003b).

Relações similares ocorrem em outros sistemas nos quais há mistura de gases (e.g., $\left.\mathrm{H}_{2} \mathrm{O}-\mathrm{CO}_{2}-\mathrm{N}_{2}\right)$. A identificação prévia das espécies gasosas utilizando outros métodos auxiliares, como a microespectrometria a laser Raman (e.g., Frezzotti et al. 2012) ou a análise de voláteis (e.g., Salvi \& Williams-Jones 2003), é indispensável na discriminação e estimativa das proporções de fases gasosas.

\subsection{Exemplos de Sistemas Químicos de Inclusões Fluidas Aquosas}

Similar à determinação da composição do sistema químico das inclusões fluidas aquosas, a estimativa da salinidade das soluções eletrolíticas é realizada a partir do conhecimento do diagrama de fases que rege os pontos de fusão de fases quanto à variação de salinidade do fluido, geralmente de acordo com uma curva cotética (Roedder 1984, Bodnar 2003c).

Três sistemas químicos são mais comumente identificados nas soluções aquosas eletrolíticas que ocorrem em ambientes geológicos (Bodnar 2003b): (a) o sistema $\mathrm{NaCl}-\mathrm{H}_{2} \mathrm{O}$; (b) o sistema $\mathrm{CaCl}_{2}-\mathrm{NaCl}-\mathrm{H}_{2} \mathrm{O}$; e (c) o sistema KCl-NaCl-H $\mathrm{H}_{2} \mathrm{O}$.

Também é comum a presença de inclusões portadoras de gás representadas por sistemas químicos do tipo (d) SAL-GÁS- $\mathrm{H}_{2} \mathrm{O}$, onde a fração gasosa é geralmente composta por $\mathrm{CO}_{2}$ puro ou associado a outro gás (e.g., $\mathrm{CH}_{4}$, etc.), e a fração aquosa compreende um subsistema químico referente aos três sis- temas anteriormente descritos (Diamond 2003b).

Uma breve introdução sobre o comportamento de cada um desses sistemas e sua relação com a estimativa da salinidade é mostrada abaixo:

\section{(a) Sistema NaCl- $\mathrm{H}_{2} \mathrm{O}$}

No sistema químico binário composto de $\mathrm{NaCl}-\mathrm{H}_{2} \mathrm{O}$, o diagrama de fases (Fig. 9) que rege o comportamento em baixa temperatura da solução indica que, para inclusões aquosas com salinidades inferiores a 23,2\% em massa de $\mathrm{NaCl}^{2}$, a primeira fase a fundir-se na temperatura do eutético estável (i.e., $-21,2^{\circ} \mathrm{C}$ ) é a hidrohalita. Essas condições variam com relação à temperatura de fusão do gelo (Hall et al. 1988, Bodnar 1993, Bodnar 2003b). Equações empíricas como a apresentada por Bodnar (1993) podem ser utilizadas para calcular a salinidade desses fluidos nessas circunstâncias.

Em salinidades superiores a 23,2 e inferiores a $26,3 \%$ em massa de $\mathrm{NaCl}$, o gelo é a primeira fase a fundir-se completamente na temperatura do eutético estável. Nesse caso, a hidrohalita permanece estável até sua fusão final de acordo com a curva cotética proporcional à salinidade da solução (Bodnar 2003b). A relação utilizada para a determinação da salinidade nesta composição é apresentada por Sterner et al. $(1988,1992)$ como subsistema do sistema NaCl-KCl- $\mathrm{H}_{2} \mathrm{O}$. É importante ressaltar que inclusões formadas com essas salinidades são raramente descritas na literatura, em parte devido à dificuldade do reconhecimento da hidrohalita como última fase a fundir-se durante a análise microtermométrica (Bodnar 2003b).

Nos casos em que a solução aquosa apresenta valores de salinidade superiores a $26,3 \%$ em massa de $\mathrm{NaCl}$, a última fase sólida acima do ponto peritético é a halita (Bodnar 2003c). Na prática, contudo, inclusões com valores de salinidade entre 30-35\% em massa de $\mathrm{NaCl}$ não precipitam halita (Bodnar 2003c). Nessas condições a salinidade das inclusões fluidas não pode ser calculada pela fusão final de gelo ou da hidrohalita (Bodnar 2003b).

Modelos para o cálculo das salinidades deste tipo de inclusão foram construídos utilizando-se a temperatura de dissolução da halita (Sterner et al. 1988, Bodnar 1994). Foram considerados os casos

$2 \mathrm{Na}$ literatura em geral sobre inclusões fluidas utiliza-se tradicionalmente a medida de concentração em "percentagem em peso equivalente". 0 peso é a força exercida sobre os corpos pela ação do campo gravitacional da Terra e, portanto, depende da aceleração da gravidade. A estimativa composicional que é feita pelos parâmetros de inclusões fluidas, no entanto, não depende da aceleração da gravidade (Bakker 2011). 


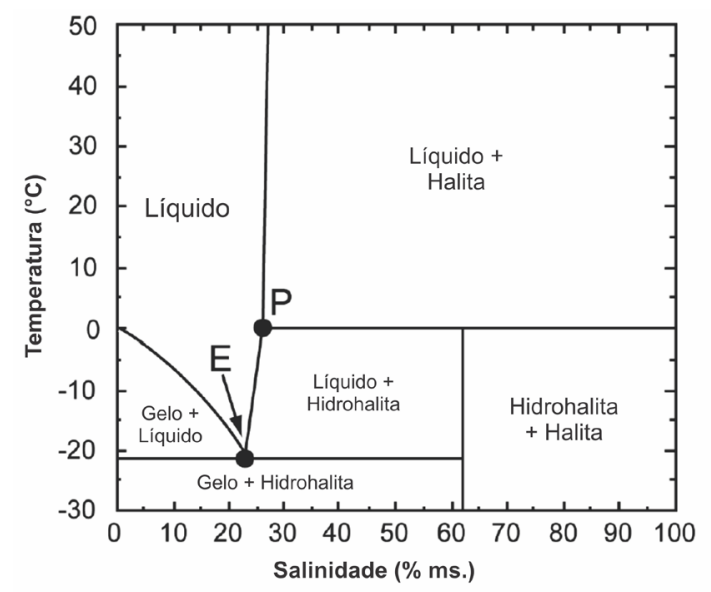

rigura y. Uiagrama de tases do sistema bınarıo INaCI- $\mathrm{H}_{2} \mathrm{U}$ (Hall et al. 1988, Sterner et al. 1988, Bodnar et al. 1989, Bodnar 2003b).

em que a dissolução da halita ocorre ainda com a presença da fase vapor (Sterner et al. 1988), ou a dissolução da halita compreende a última fase a homogeneizar-se (Bodnar 1994).

Bakker (2012a) propôs um novo modelo, argumentando que o modelo de Sterner et al. (1992) fornece resultados de salinidade superestimados, visto que a fase vapor ainda conteria quantidades significativas de água. Ainda mais, Bakker (2012b) aponta que este modelo não respeita a regra de fases de Gibbs, pois para um sistema com três componentes seriam necessários dois parâmetros para a obtenção da composição. Steele-MacInnis \& Bodnar (2013), em contrapartida, reformularam o modelo e com base em estudos empíricos mostraram que o erro do cálculo da salinidade pela dissolução da halita ainda na presença de fase vapor é geralmente menor que $\sim 1,5 \%$ em massa de $\mathrm{NaCl}$.

\section{(a) Sistema $\mathrm{KCl}-\mathrm{NaCl}-\mathrm{H} 2 \mathrm{O}$}

Em sistemas magmático-hidrotermais associados aos magmas graníticos, os cátions dominantes no meio aquoso são o Na e o K (Burnham 1997). Em especial, inclusões fluidas contendo minerais filhos de silvita e halita são típicos de depósitos de cobre tipo pórfiro (Roedder \& Bodnar 1997). Também são comuns em bacias evaporíticas (e.g., Sabouraud 1994) e podem estar relacionados à diagênese (e.g., Goldstein \& Reynolds 1994).

$\mathrm{O}$ diagrama do sistema KCl-NaCl- $\mathrm{H}_{2} \mathrm{O}$ (Fig. 10 ) indica a existência de inclusões saturadas e sub-saturadas em sais (Bodnar 2003b). Dependendo da composição da inclusão fluida deste sistema, 22 possibilidades de combinações de mudanças de fase podem acontecer (Sterner et al. 1988). Sterner et al. (1988) demonstraram que a salinidade pode ser determinada a partir da temperatura de dissolução da última fase sólida, halita ou silvita. No caso no qual a última fase a ser dissolvida é a halita, utiliza-se a mesma equação para soluções supersaturadas no sistema $\mathrm{NaCl}-\mathrm{H}_{2} \mathrm{O}$. Caso contrário, utiliza-se uma equação com base na temperatura de dissolução da silvita (Sterner et al. 1988).

Refutando a acurácia do modelo de cálculo de salinidade com base exclusivamente na temperatura de dissolução da halita e silvita, Bakker (2012b) propôs um novo modelo empírico para o cálculo de salinidade para o sistema $\mathrm{KCl}-\mathrm{NaCl}-\mathrm{H}_{2} \mathrm{O}$, à similaridade do modelo proposto para o sistema $\mathrm{NaCl}-\mathrm{H}_{2} \mathrm{O}$ (Bakker 2012a).

\section{(a) Sistema CaCl2-NaCl-H2O}

Fluidos com composição química do sistema $\mathrm{CaCl}_{2}-\mathrm{NaCl}-\mathrm{H}_{2} \mathrm{O}$ são abundantes em diversos ambientes geológicos, sejam em bacias sedimentares (e.g., Collins 1975, Hanor \& McIntosh 2007), em ambientes hidrotermais submarinos (e.g., Vanko 1988, Vanko et al. 1988) e em diversas classes de depósitos minerais, a exemplo: depósitos de $\mathrm{Pb}-\mathrm{Zn}$ do tipo Mississippi Valley (e.g., Haynes \& Kesler 1987, Basuki \& Spooner 2002, Stoffell et al. 2008), depósitos de ouro orogênico (e.g., Robert \& Kelly 1987), depósitos de óxido de ferro-Cu-Au, também denominados pela sigla IOCG (e.g., Xu 2000).

As inclusões fluidas deste sistema (Fig. 11) que apresentam apenas as fases vapor e líquida à temperatura ambiente, quando completamente congeladas apresentam três fases sólidas: gelo, hidrohalita $\left(\mathrm{NaCl} \cdot 2 \mathrm{H}_{2} \mathrm{O}\right)$ e antarticita $\left(\mathrm{CaCl}_{2} \cdot 6 \mathrm{H}_{2} \mathrm{O}\right)$. Durante o aquecimento, usualmente a primeira fase a fundir-se é a antarticita na temperatura do

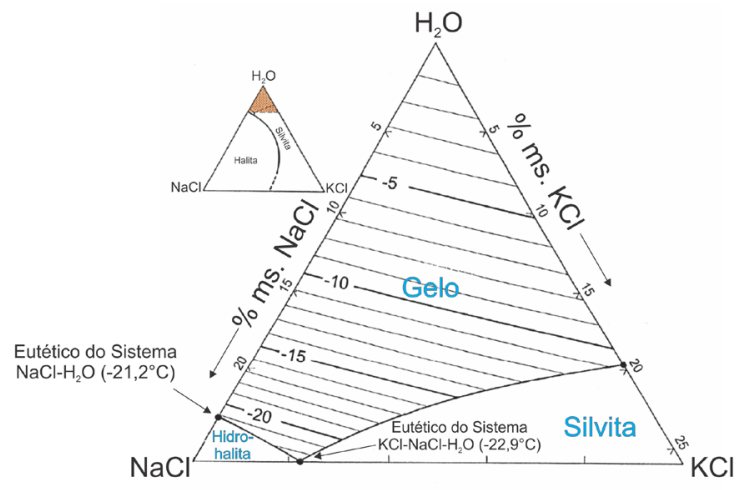

Figura 10. Diagrama de fases do sistema $\mathrm{KCl}-\mathrm{NaCl}-\mathrm{H}_{2} \mathrm{O}$ em baixa temperatura (Hall et al. 1988, Bodnar 2003b). 


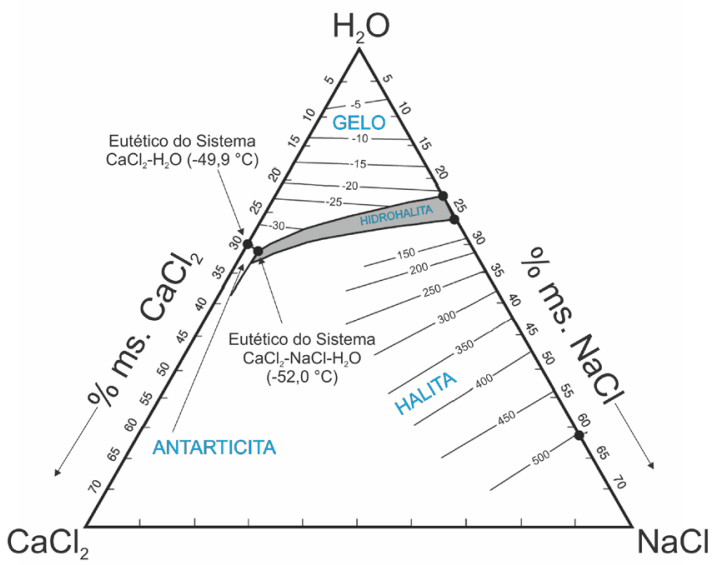

Figura 11. Diagrama de fases do sistema $\mathrm{CaCl}_{2}-\mathrm{NaCl}_{-} \mathrm{H}_{2} \mathrm{O}$ (Vanko et al. 1988, Bodnar 2003b).

eutético estável, seguindo-se a fusão da hidrohalita e, posteriormente, do gelo (Bodnar 2003b).

Em fluidos supersaturados, a halita ocorre como mineral filho a temperatura ambiente. Já em inclusões fluidas com composições excepcionalmente ricas em cálcio, além de halita ocorre em também a tetrahidrato $\left(\mathrm{CaCl}_{2} \cdot 2 \mathrm{H}_{2} \mathrm{O}\right)$ ou mesmo dihidrato $\left(\mathrm{CaCl}_{2} \cdot 4 \mathrm{H}_{2} \mathrm{O}\right)$ como mineral filho estável (Steele-MacInnis et al. 2011).

Diferentes modelos para o cálculo da composição deste tipo de fluido são encontrados na literatura utilizando dados empíricos e teóricos (Vanko et al. 1988, Oakes et al. 1990, Williams-Jones \& Samson 1990, Naden 1996, Chi \& Ni 2007).

O modelo mais recente (Steele-MacInnis et al. 2011) foi baseado em dados microtermométricos e microanalíticos e permite o cálculo da composição de inclusões fluidas do sistema $\mathrm{CaCl}_{2}-\mathrm{NaCl}-\mathrm{H}_{2} \mathrm{O}$ com base em duas propriedades de mudança de fase, baseado no ponto de fusão de dois sólidos (e.g., hidrohalita e gelo para inclusões subsaturadas, ou halita e gelo, para composição saturadas e ricas em sódio, etc.). Este modelo, em particular, inclui inclusive as composições ricas em cálcio onde se prevê a possibilidade da presença de tetrahidrato e dihidrato de cálcio como minerais filhos (Steele-MacInnis et al. 2011).

\subsection{As Inclusões Fluidas de Hidrocarbonetos}

Uma classe de inclusões fluidas formadas majoritariamente em ambiente sedimentar são as inclusões fluidas portadoras de hidrocarbonetos associados a sistemas petrolíferos (Burruss 1981, Munz 2001, Burruss 2003). Essas inclusões fluidas compreendem tanto aquelas inclusões fluidas de óleo como as inclusões fluidas de gás associado a um sistema petrolífero, portadoras exclusivamente de hidrocarbonetos leves (i.e., $\mathrm{C}_{1}-\mathrm{C}_{5}$; Burruss 2003).

$\mathrm{Na}$ literatura em geral, as inclusões fluidas de óleo são também denominadas na literatura como inclusões fluidas de petróleo (e.g., Roedder 1984, Munz 2001, Burruss 2003), inclusões fluidas de hidrocarbonetos (e.g., Burruss 1981), ou simplesmente chamadas por alguns autores de inclusões de óleo (e.g., Eadinton \& Kempton 2008) dependendo da técnica e dos objetivos de estudo.

As inclusões fluidas de óleo, ao contrário das inclusões portadoras exclusivamente das fases gasosas, ocorrem principalmente em bacias sedimentares (Munz 2001) e raramente são encontradas em rochas metamórficas e ígneas fraturadas (e.g., Munz et al. 2002). Diferentemente das inclusões compostas apenas por gases, as inclusões de óleo são prontamente reconhecidas por meio de microscopia, pois as mesmas fluorescem quando excitadas pela luz ultravioleta (Fig. 12) emitindo luz no comprimento de onda visível ao olho humano (Burruss 1981).

Como são portadoras da fração mais pesada dos hidrocarbonetos, essas inclusões têm composição complexa (Fig. 13) compreendida desde as frações mais leves (gases de hidrocarbonetos)
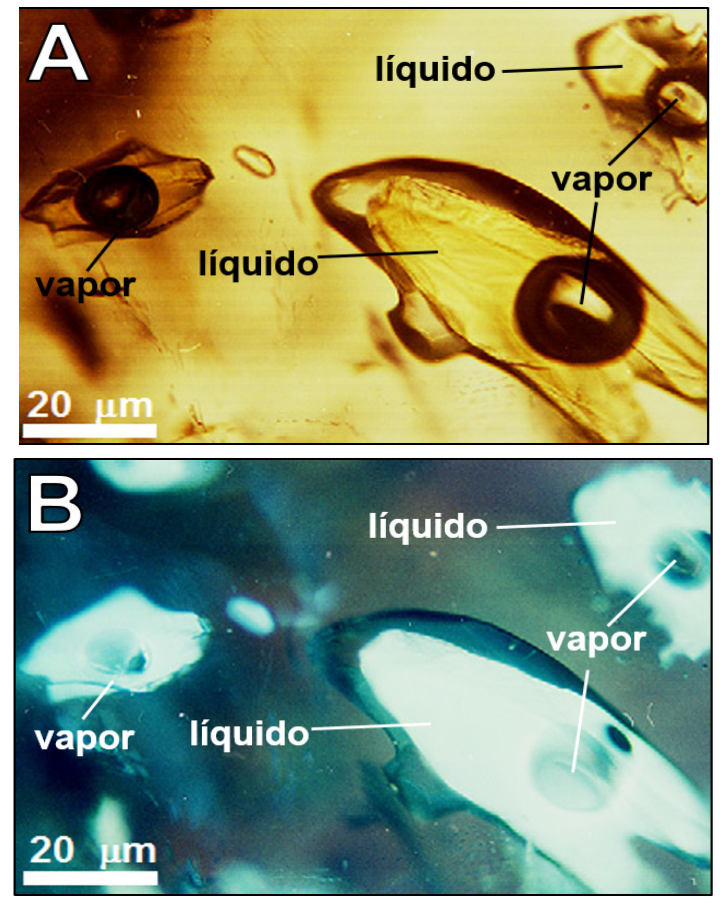

Figura 12. Fotomicrografia de exemplos de inclusões fluidas de óleo em (A) luz transmitida polarizada branca e (B) sob luz ultravioleta epifluorescente com cor de fluorescência branca azulada (Suchý et al. 2010). 


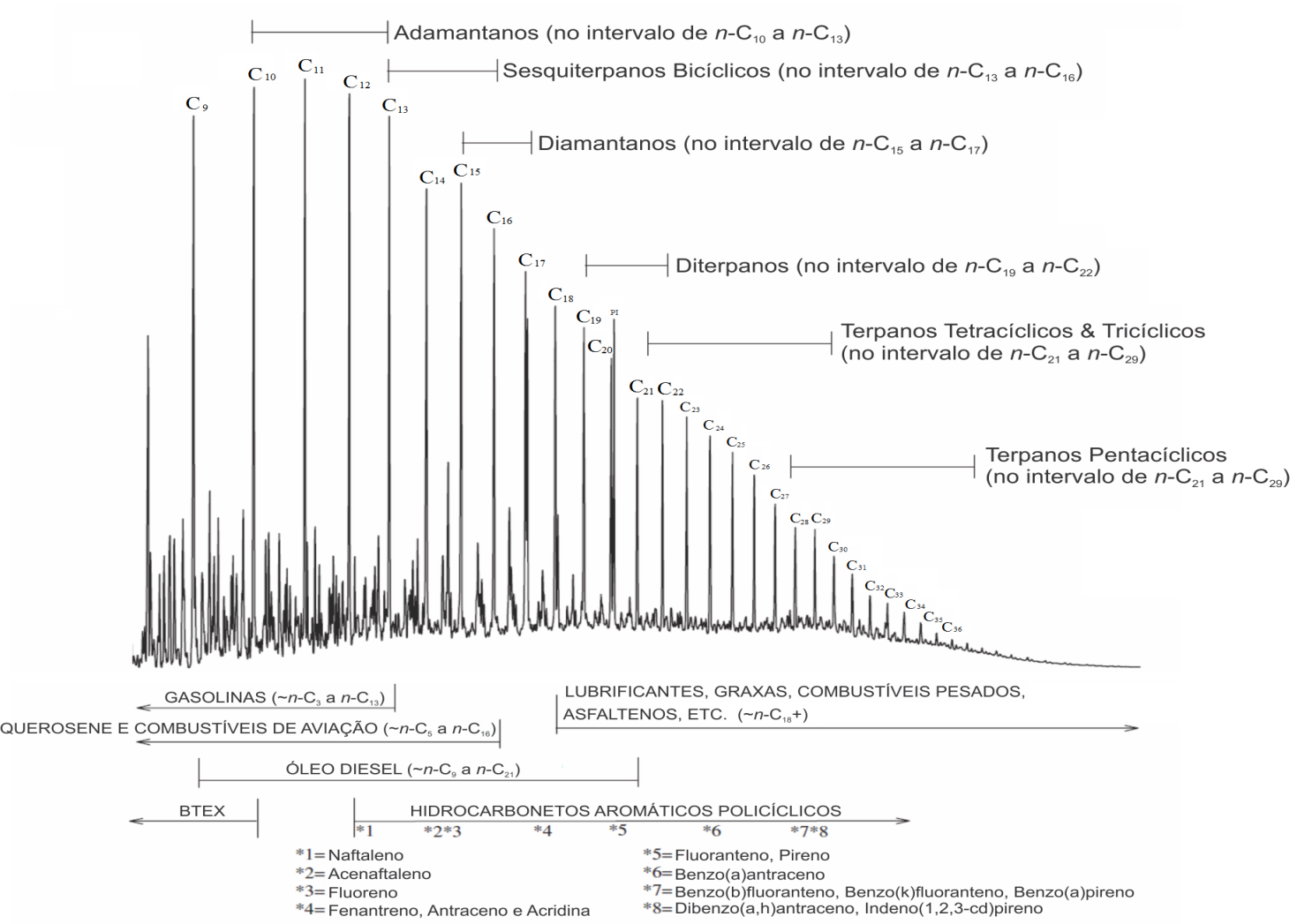

Figura 13. Cromatograma em fase gasosa de petróleo, que exemplifica toda a abrangência de classes de componentes presentes no petróleo de uma inclusão fluida, incluindo compostos saturados, compostos aromáticos (BTEX-benzeno, tolueno, etil-benzeno, xileno e hidrocarbonetos aromáticos policíclicos) e diferentes classes de biomarcadores (diamantoides, terpanos, etc.), além de resinas e asfaltenos não resolvidos por esse tipo de análise (modificado de Wang et al. 2007).

até as frações mais pesadas dos óleos que incluem toda a classe de compostos saturados parafínicos, além de compostos aromáticos, resinas e asfaltenos (Burruss 2003). Ainda em menor proporção, essas inclusões fluidas também apresentam fósseis moleculares (biomarcadores) reconhecíveis com análises laboratoriais (Burruss 2003).

Uma grande abundância de inclusões fluidas de óleo está geralmente associada ao registro ubíquo da atividade de um sistema petrolífero, podendo estar presentes em paleorotas de migração, em grandes perfis verticais em reservatórios de petróleo atuais, ou mesmo aqueles exauridos, por exemplo, devido ao rompimento parcial ou total da rocha selante (CSIRO 1996, Munz 2001, George et al. 2004, George et al. 2007). Uma vez formadas, as inclusões de óleo constituem sistemas fechados preservando o óleo de qualquer modificação posterior ao preenchimento do reservatório. Por isso, alguns autores interpretam o óleo das inclusões fluidas como um paleo-óleo (e.g., Lisk \& Eadington 1994, Pan et al. 2003, George et al. 2004), representativo dos primeiros pulsos de migração para o reservatório. Por conta disso, diversos autores aplicaram estes conceitos para interpretar as condições prevalecentes no momento inicial de preenchimento dos reservatórios (e.g., Lisk et al. 1998, Parnell et al. 2001, Lisk et al. 2012, Xie et al. 2013).

A formação das inclusões de óleo e de gás depende de uma série de fatores como o favorecimento da cimentação, a permo-porosidade, a saturação de óleo e a molhabilidade do reservatório (Munz 2001, Teinturier \& Pironon 2004). Durante os estágios iniciais de preenchimento de um reservatório, as rochas apresentam saturação de óleo variável o que permite que a cimentação dos poros e a recristalização de microfraturas formem inclusões fluidas (England et al. 1987, Munz 2001). Como o fluido depende de espaço para percolar pela rocha, a formação de inclusões fluidas depende de boa permo-porosidade (Goldstein \& Reynolds 1994), a exemplo do campo de Ula, no Mar do Norte (Oxby et al. 1995).

Com relação à saturação de fluidos e à molhabilidade, em estudo experimental, Teinturier \& 
Pironon (2004) demonstraram a formação de inclusões fluidas de óleo e aquosas a partir da recristalização de microfraturas e em sobrecrescimento de cristais de quartzo para diferentes proporções de água/óleo. Os resultados mostraram que a formação das inclusões fluidas é dependente da molhabilidade dos grãos a óleo ou a água, tanto quanto a proporção de água e óleo do sistema (Teinturier \& Pironon 2004). Quando o meio se encontra molhado a óleo, a formação de inclusões fluidas é rara (Teinturier \& Pironon 2004). Quando o meio se encontra molhado a água, a formação de inclusões fluidas ocorre até mesmo quando o reservatório se encontra quase saturado em óleo (i.e., 90 \% em massa de óleo e $10 \%$ em massa de água; Teinturier \& Pironon 2004). Por outro lado, a segregação de óleo e água previamente à formação das inclusões fluidas pode inibir a formação de inclusões aquosas saturadas em metano (Teinturier \& Pironon 2004). Experimentos similares feitos com calcita mostraram que é possível a formação de inclusões fluidas de óleo em microfraturas recristalizadas em reservatórios saturados em óleo (Chen et al. 2015).

Ainda quanto aos experimentos de formação de inclusões sintéticas, o óleo aprisionado nas inclusões é representativo quimicamente do óleo parental, em termos da composição de saturados, aromáticos, resinas e asfaltenos até $250{ }^{\circ} \mathrm{C}$ para inclusões hospedadas em quartzo, muito embora possa haver inversão na proporção de resinas e asfaltenos (Teinturier et al. 2003). Craqueamento térmico com geração de metano foi observado a temperaturas acima de $250{ }^{\circ} \mathrm{C}$ (Teinturier et al. 2003).

O modo de aprisionamento das inclusões fluidas afeta de modo significativo a composição das inclusões em termos de saturados, aromáticos, resinas e asfaltenos (Pang et al. 1998). Em estudos cromatográficos de óleos de inclusões fluidas e de óleo cru provenientes de diferentes bacias da Austrália e de Papua Nova Guiné, Pang et al. (1998) revelaram que os óleos aprisionados em inclusões fluidas que se formaram junto ao cimento diagenético exibem um enriquecimento em compostos aromáticos, resinas e asfaltenos em comparação com o óleo do reservatório. O mesmo não foi observado para os óleos das inclusões fluidas formadas em microfraturas, que mostraram composição próxima a dos óleos dos reservatórios (Pang et al. 1998).

\subsubsection{Termodinâmica do Petróleo}

Diferentemente das inclusões fluidas aquosas que compreendem sistemas químicos simples (Bodnar 2003b, Diamond 2003b), cuja composição global pode ser facilmente estimada a partir de métodos indiretos como a microtermometria (Roedder 1984) permitindo a reconstrução do campo de estabilidade termodinâmico de cada fase fluida a partir das equações de estado simplificadas (e.g., Bodnar \& Vityk 1994, Atkinson 2002, Bakker \& Brown 2003, etc.), os óleos minerais consistem de uma complexa mistura de compostos orgânicos, o que não permite uma simples estimativa composicional via análise indireta (Munz 2001, Burruss 2003).

Como exemplo, diferentes classificações de óleo com base na sua composição podem ser encontradas na literatura dependendo da aplicação (McCain 1990). Na geoquímica orgânica, usualmente os óleos são classificados quanto ao paleoambiente deposicional das respectivas rochas geradoras (e.g., óleos marinhos, lacustres, de ambiente transicional evaporítico, etc.) e à maturidade térmica durante a geração (Tissot \& Welte 1984). Contudo, como o estudo das inclusões fluidas volta-se para a paleotermometria e paleobarometria, faz-se necessário também o uso de classificações físico-químicas do petróleo (Munz 2001).

As acumulações de petróleo são comumente classificadas como acumulações de (1) gás natural, de (2) condensados, de (3) óleo volátil e de (4) black oil (Fig. 14; Munz 2001). Essa variação na composição deriva da proporção dos maiores componentes presentes no petróleo, ou seja, a proporção de hidrocarbonetos leves aumenta na seguinte ordem: black oil, óleo volátil, condensados e gás natural (McCain 1990). O gás natural pode ainda ser classificado como gás seco ou gás úmido (McCain 1990). O gás seco é constituído quase que exclusivamente por metano, com traços de etano, propano, butano, nitrogênio e pentano, além de poder conter outros gases que não são hidrocarbonetos, como o dióxido de carbono (McCain 1990). Já o gás úmido mostra a presença dos hidrocarbonetos leves $\left(\mathrm{C}_{1}-\mathrm{C}_{5}\right)$ em associação com traços de hidrocarbonetos mais pesados $\left(\mathrm{C}_{6}-\mathrm{C}_{7+}\right)$, além de poder apresentar ou não outros compostos constituídos de moléculas inorgânicas (McCain 1990).

Tal variação é derivada do estágio de maturação térmica da rocha geradora e implica em diferentes características termodinâmicas (Tissot \& Welte 1984). Seguindo o exemplo dado por Munz (2001; Fig. 14), em um reservatório de gás seco, por exem- 


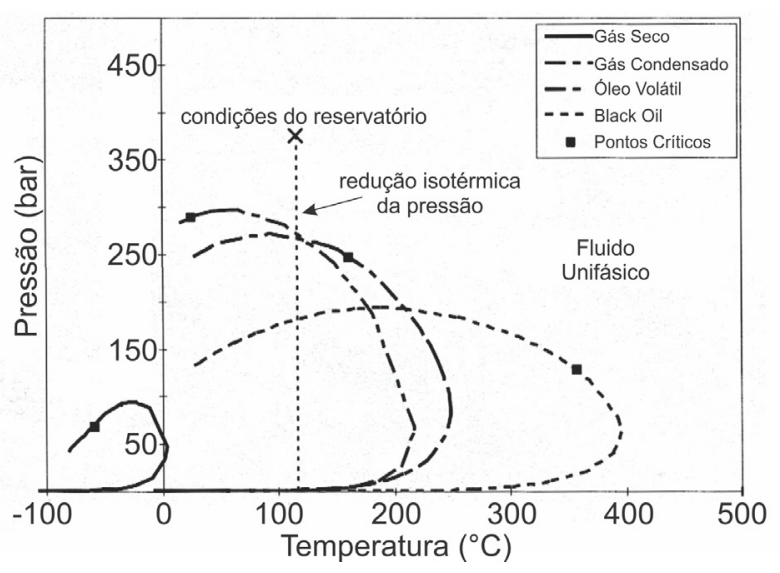

Figura 14. Diagrama P-T mostrando as isopletas representativas das diferentes classes de petróleo (Munz 2001).

plo, a redução de pressão isotérmica em condições naturais de temperatura nunca causará a dissociação do fluido em duas fases, o que no diagrama de pressão e temperatura corresponde à curva dos líquidos.

Por outro lado, a redução de pressão isotérmica em um reservatório de condensado faz com que a mudança de fase ocorra no ponto de orvalho, ou seja, ocorre a condensação de parte da fase vapor em líquido (Munz 2001). Reservatórios de óleo volátil e black oil, quando submetidos à redução de pressão isotérmica, dissociam-se em duas fases a partir do cruzamento com o ponto de bolha, caracterizado pela evaporação de parte da fração líquida (Munz 2001).

A partir da composição molar percentual do petróleo e de equações de estado são feitos os cálculos de isopletas e isócoras utilizados na predição de características físico-químicas e em última instância na interpretação paleotermométrica e paleobarométrica das inclusões fluidas de óleo e de gás associado ao petróleo (Munz 2001). Os resultados mais acurados para os cálculos de simulação do comportamento termodinâmico de petróleo são conseguidos a partir do uso de equações de estado cúbicas (McCain 1990), como as equações de Soave-Redlich-Kwong (Soave 1972) e Peng-Robinson (Peng \& Robinson 1976).

A limitação na capacidade de estimativa da composição molar percentual de cada componente para as pequenas alíquotas de óleo de uma inclusão fluida restringe a aplicação direta de equações de estado (Thiéry et al. 2002). É possível fazer uma inferência da composição global dos óleos a partir da fração de vapor (Fv) e do valor de Th (Fig. 15) das inclusões fluidas (Bourdet et al. 2008).

Contudo, este método é limitado às condições de contorno experimentais e deve ser utilizado apenas como um indicativo composicional e na identificação de fenômenos de reequilíbrio (Fig. 16) das inclusões fluidas devido a modificação pós-aprisionamento (Bourdet et al. 2008).

$\mathrm{Na}$ literatura o problema da estimativa composicional foi parcialmente contornado de duas maneiras diferentes: (i) assumindo-se a composição do óleo das inclusões equivalente àquela do óleo cru atualmente presente no reservatório (e.g., Narr \& Burruss 1984, McLimans 1987, Bodnar 1990) ou (ii) pela estimativa da composição a partir de um modelo pseudo-composicional (e.g., Aplin et al. 1999, Thiéry et al. 2002).

No primeiro caso, a composição é assumida arbitrariamente como a mesma do reservatório com parâmetros indiretos (e.g., reservatórios de idade geológica recente; similaridades entre parâmetros composicionais estimados do óleo das inclusões e do óleo do reservatório) e a mesma é aplicada em equações de estado conhecidas (Munz 2001). Contudo, essa abordagem é limitada visto que vários reservatórios de petróleo apresentam modificações químicas significativas posteriores à entrada do óleo no reservatório (Aplin et al. 1999, Thiéry et al. 2002), como processos de biodegradação, lavagem do óleo por influxo de água (water-washing) ou de gás (gas-washing) e mistura de diferentes tipos de óleo (Tissot \& Welte 1984).

No segundo caso, uma pseudo-composição é formulada a partir de processos matemáticos iterativos, seja a partir da composição de um óleo com contexto geológico similar (e.g., Aplin et al. 1999) ou a partir da inferência realizada exclusivamente a partir de parâmetros físico-químicos do óleo das inclusões fluidas com base em uma biblioteca de variadas composições de óleo (e.g., Thiéry et al. 2002).

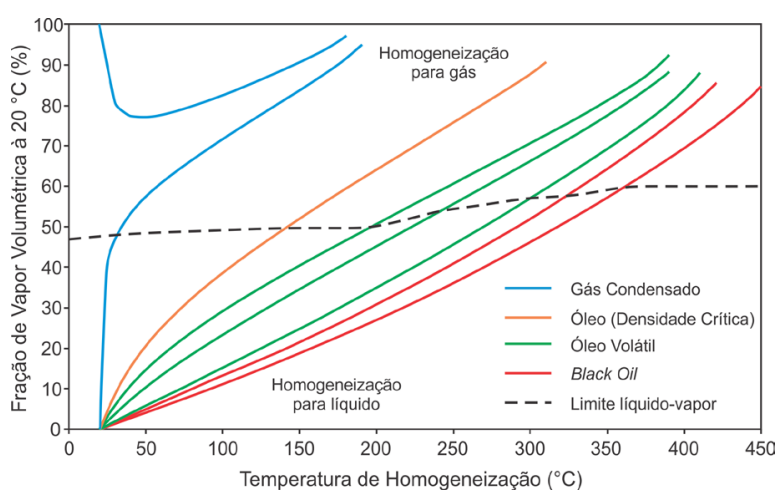

Figura 15. Diagrama Th versus Fv a $20^{\circ} \mathrm{C}$, com os campos de ocorrência das principais classes de petróleo (McCain 1990) para inclusões fluidas de petróleo teóricas (simplificado de Bourdet et al. 2008). 


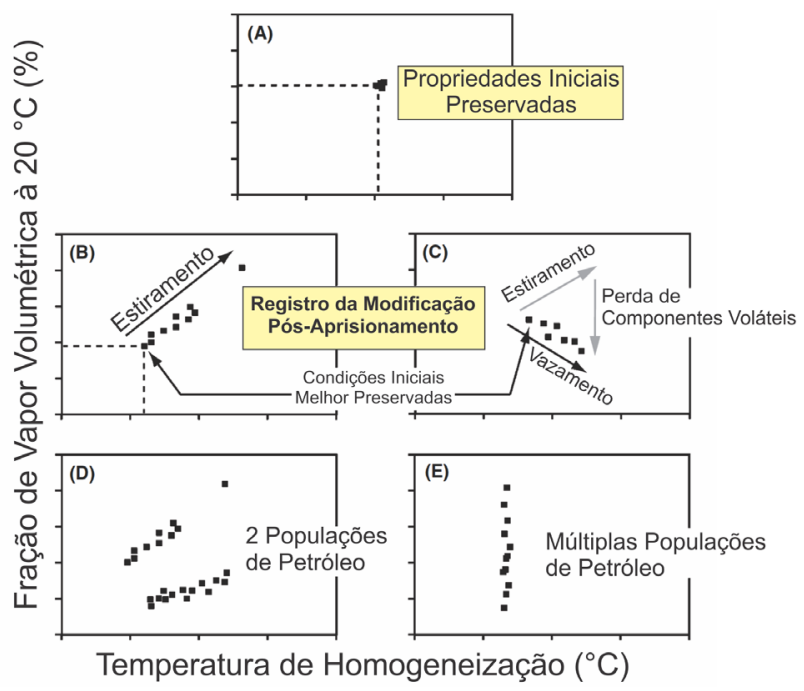

Figura 16. Diagrama esquemático de Th versus Fv mostrando as direções de variação de valores correspondentes ao esperado para inclusões de petróleo que exibem modificação pós-aprisionamento ou aprisionamento de diferentes populações de petróleo (Bourdet et al. 2008).

O método de Aplin et al. (1999) consiste do uso da composição do óleo atualmente presente no reservatório como parâmetro de entrada de um modelo matemático de regressão com uso de softwares comerciais voltados para simulação PVT (Pressão-Volume-Temperatura). O processo iterativo baseia-se no valor de Th medido na microtermometria e de Fv medido a partir de microscopia confocal de varredura a laser (Aplin et al. 1999). A maior limitação deste método está na grande dependência do petróleo inicial escolhido (Aplin et al. 1999).

No método de Aplin et al. (1999) são seguidos 8 passos, implementados como um algoritmo no software comercial VTFlinc(C) (VTFlinc 1997) de modo interativo para estimar a composição do óleo das inclusões:

(i) no primeiro passo, escolhe-se a composição de um petróleo de um reservatório próximo ou com mesmas características 'genéticas' do óleo das inclusões fluidas;

(ii) no segundo passo, a partir do software de simulação PVT é calculada a pressão de saturação na temperatura de saturação (i.e., temperatura de homogeneização) da inclusão fluida;

(iii) no terceiro passo realiza-se uma simulação 'flash' ou cálculo de composição de fases na temperatura de saturação, notando o volume molar total de petróleo; (iv) no quarto passo calcula-se a pressão de saturação do petróleo a temperatura ambiente. Essa deve ser a máxima pressão dentro da inclusão fluida a temperatura ambiente;

no quinto passo é realizada uma série de simulações flash' ou de cálculo de composição de fases a temperatura ambiente e para diferentes valores de pressão abaixo da pressão de saturação. Nesta etapa são anotados os volumes molares para cada valor de pressão, sendo que quando o volume molar da mistura a temperatura ambiente equivale ao volume molar da temperatura de saturação, os dois pontos encontram-se na mesma isócora;

(v) no sexto passo é calculada a razão líquido/ vapor a temperatura ambiente do petróleo incluído e do petróleo escolhido para o início da simulação. Se o volume calculado para as fases líquida e vapor equivale ao volume medido pela microscopia confocal de varredura a laser, então a composição do petróleo inicial é considerada uma representação acurada para a modelagem do petróleo da inclusão fluida;

(vi) no passo sete, caso a condição do passo seis não seja atendida, uma quantidade arbitraria de gás natural é adicionado ou removido da composição do petróleo originalmente escolhida;

(vii) no passo oito o procedimento é repetido até que a condição definida no passo seis seja estabelecida.

Thiéry et al. (2000, 2002) desenvolveram o modelo $\alpha-\beta$ baseado no trabalho de Montel (1993) e o implementaram no software FIT-Oil@ (Thiéry 2011). Neste modelo, a composição do petróleo pode ser simplificada por uma expressão com base em duas variáveis (Eq. 1).

$$
x_{n+1}=\alpha *\left(1-\frac{\beta}{n+1}\right) * x_{n}(\text { Equação } 1)
$$

Onde $x_{n} e_{n+1}$ correspondem às frações molares dos cortes com " $n$ " $\mathrm{e}$ " $n+1$ " átomos de carbono e " $\alpha$ " e " $\beta$ " são parâmetros obtidos a partir da regressão matemática da composição de petróleos de um banco de dados (Thiéry et al. 2002, Ping et al. 2011). O parâmetro " $\alpha$ " descreve a distribuição geométrica das frações não voláteis (i.e., com " $\mathrm{n}$ " maior que 10) como função de "n", com valores típicos entre 0,6 e 0,91 (Ping et al. 2011). Já o parâmetro " $\beta$ " descreve a variação na composição da fração volátil (i.e., de $\mathrm{C}_{1}-\mathrm{C}_{6}$ ), indicando o seu enriquecimento para valores positivos ou o seu 
empobrecimento em voláteis para valores negativos, sendo que valores típicos ocorrem entre $-0,2$ e 1 (Ping et al. 2011). Nota-se aqui que na prática é utilizada uma versão modificada da equação 4.1, que pode ser encontrada no apêndice de Thiéry et al. (2002) e é a versão disponibilizada junto ao software FIT-OIL@ (Thiéry 2011).

O processo de cálculo da curva " $\alpha-\beta$ " compreende um modelo iterativo entre os parâmetros composicionais da inclusão fluida e os valores de " $\alpha$ " e " $\beta$ " (Thiéry 2011). Para calcular um ponto " $\alpha-\beta$ " considera-se as propriedades isocóricas de uma inclusão fluida, onde para um dado conjunto dos valores de Fv medido pela microscopia confocal por varredura a laser, temperatura de medição (Tv) da Fv e Th, nós calculamos " $\alpha$ " em função de " $\beta$ " primeiramente assumindo um valor para " $\alpha$ " (e.g., $0,9)$, e em seguida calculamos o volume total $\left(\mathrm{V}_{1}\right)$ para Th (Thiéry 2011). Então, calcula-se novamente o volume total $\left(\mathrm{V}_{2}\right)$, mas dessa vez a partir de mesma composição assumida de óleo, considerando o valor de Fv e a temperatura de medição Tv (Thiéry 2011). O procedimento é repetido até os valores de $V_{1}$ e $V_{2}$ sejam equivalentes (Thiéry 2011). Uma vantagem do modelo " $\alpha-\beta$ ” é a representação de diferentes composições de petróleo em um diagrama bivariante (Fig. 17; Thiéry et al. 2012), que é similar a comparação direta entre $\mathrm{Fv}$ e Th (Bourdet et al. 2008).

O modelo " $\alpha-\beta$ " é limitado, pois apenas com os parâmetros de Th, Fv e Tv não se pode limitar um único par de valores " $\alpha$ " e " $\beta$ ”, sendo necessário estipular outro parâmetro de contorno, seja pelo

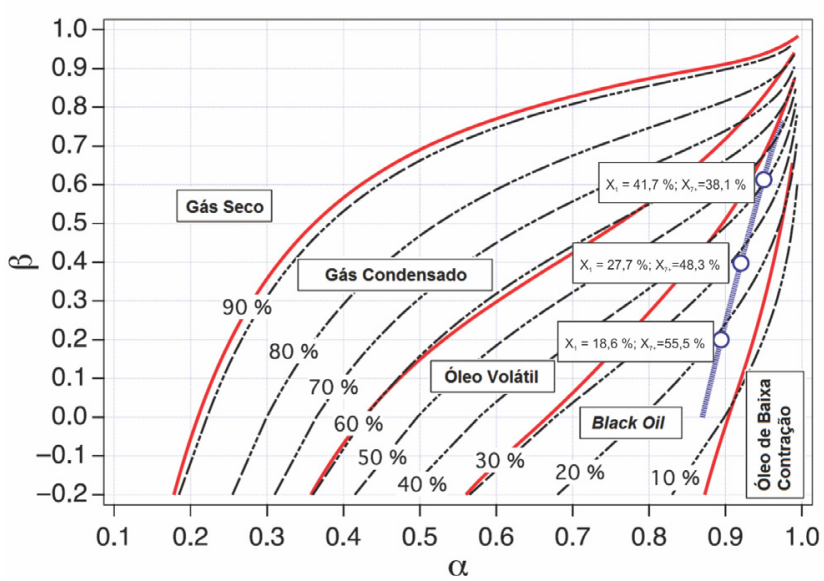

Figura 17. Diagrama " $\alpha-\beta$ " com os campos de valores para as principais classes composicionais de petróleo (McCain 1990) limitado em vermelho e exemplo de curva $\alpha(\beta)$ em roxo. Valores da composição molar percentual de metano $\left(C_{1}\right)$ são indicados nas curvas tracejadas em preto (Ping et al. 2011).
'API, que pode ser estimada de modo indireto (e.g., Stasiuk \& Snowdon 1997), ou pela estimativa da percentagem molar de metano a partir da microscopia FT-IR (e.g., Pironon et al. 2001).

Posteriormente, Ping et al. (2011) apresentaram um modelo " $\alpha-\beta$ " simplificado, denominado como modelo " $\beta$ ". Este modelo não se encontra implementado no software FIT-Oil@ (Thiéry 2011). Esta simplificação foi realizada com uma nova interpolação dos parâmetros composicionais dos petróleos fixando um valor de " $\alpha$ " (i.e., 0,92), já que o intervalo de variação de " $\alpha$ " é consideravelmente pequeno em comparação com " $\beta$ " (Ping et al. 2011). A principal vantagem dessa simplificação foi poder expressar o modelo composicional com apenas um parâmetro $(\beta)$, em especial devido à correlação desse parâmetro com a composição de metano do petróleo (Ping et al. 2011).

É interessante notar que ambos os modelos de Aplin et al. (1999), Thiéry et al. (2000, 2002) e mesmo o modelo simplificado de Ping et al. (2011) apresentam vantagens e desvantagens similares (Ping et al. 2011). Em especial ambos os modelos são limitados, pois não permitem uma estimativa precisa da fração leve dos hidrocarbonetos, composta majoritariamente de metano, muito embora as estimativas permitam prever a pressão de saturação com erro de menos de $10 \%$ de desvio absoluto médio (Ping et al. 2011).

\section{Referências.}

Aplin A.C., Mcleod G., Larter S.R., Pedersen K.S., Sorensen H., Booth T. 1999. Combined use of confocal laser microscopy and PVT simulation for estimating the composition and physical properties of petroleum in fluid inclusions. Mar. Pet. Geol., 16:97-110.

Atkinson A.B. 2002. A model for the PTX properties of $\mathrm{H}_{2} \mathrm{O}-\mathrm{NaCl}$. 2002. Blacksburg: Virginia Tech. 133 p. (Dissertação de Mestrado.)

Bakker R.J. 1999. Optimal Interpretation of Microthermometrical Data from Fluid Inclusions: Thermodynamic Modelling and Computer Programming. Heidelberg: Geologisch-Paläontologisches Institut, Ruprecht-Karls-Universität Heidelberg. 54 p. (Tese de Habilitação.)

Bakker R.J. 2011. The use of quantities, units and symbols in fluid inclusion research. ECROFI, 21. Abstracts. Austria, Leoben, Montanuniversität Leoben, p. 5-11.

Bakker R.J. 2012a. Package FLUIDS. Part 4: thermodynamic modelling and purely empirical equations for $\mathrm{H}_{2} \mathrm{O}-\mathrm{NaCl}-\mathrm{KCl}$ solutions. Mineral. 
Petrol., 105:1-29.

Bakker R.J. 2012b. Can the vapour phase be neglected to estimate bulk salinity of halite bearing aqueous fluid inclusions? Cent. Eur. J. Geosci., 4(2):238-245.

Bakker R.J., Brown P.E. 2003a. Chapter 7: Computer modelling in fluid inclusion research. In: Samson I., Anderson A., Marshall D. eds. 2003. Fluid Inclusions: Analysis and Interpretation. Canadá, Ottawa: Mineral. Assoc. of Canada, p. 175-204. (Short Course Series, 32.)

Balhaus C.G., Stumpfl E.F. 1986. Sulfide and platinum mineralization in the Merensky Reef: evidence from hydrous silicates and fluid inclusions. Contrib. Mineral. Petrol., 94:193-204.

Basuki N.I., Spooner E.T.C. 2002. A review of fluid inclusion temperatures and salinities in Mississippi valley-type $\mathrm{Zn}-\mathrm{Pb}$ deposits: identifying thresholds for metal transport. Explor. Min. Geol., 11(1):1-17.

Bodnar R.J. 1990. Petroleum migration in the Miocene Monterey Formation, California, U.S.A.: Constraints from fluid-inclusion studies. Mineral. Mag., 54:295-304.

Bodnar R.J. 1993. Revised equation and table for determining the freezing point depression of $\mathrm{H}_{2} \mathrm{O}-\mathrm{NaCl}$ solutions. Geochim. Cosmochim. Acta, 57:683-684.

Bodnar R.J. 1994. Synthetic fluid inclusions. XII. Experimental determination of the liquidus and isochores for a $40 \mathrm{wt}$. \% $\mathrm{H}_{2} \mathrm{O}-\mathrm{NaCl}$ solution. $\mathrm{Ge}$ ochim. Cosmochim. Acta, 58:1053-1063.

Bodnar R.J. 2003a. Capítulo 1: Introduction to fluid inclusions. In: Samson I., Anderson A., Marshall D. eds. 2003. Fluid Inclusions: Analysis and Interpretation. Canadá, Ottawa: Mineral. Assoc. of Canada, p. 1-8. (Short Course Series, 32.)

Bodnar R.J. 2003b. Chapter 5: Introduction to Aqueous-Electrolyte Fluid Inclusions. In: Samson I., Anderson A., Marshall D. eds. 2003. Fluid Inclusions: Analysis and Interpretation. Canadá, Ottawa: Mineral. Assoc. of Canada, p. 81-100. (Short Course Series, 32.)

Bodnar R.J. 2003c. Chapter 8: Re-equilibration of fluid inclusions. In: Samson I., Anderson A., Marshall D. eds. 2003. Fluid Inclusions: Analysis and Interpretation. Canadá, Ottawa: Mineral. Assoc. of Canada, p. 213-231. (Short Course Series, 32.)

Bodnar R.J., Bethke P.M. 1984. Systematics of stretching of fluid inclusions. I. Fluorite and sphalerite at 1 atmosphere confining pressure. Econ. Geol., 79:141-161.

Bodnar R.J., Bins P.R., Hall D.L. 1989. Synthetic fluid inclusions. VI. Quantitative evaluation of the decrepitation behavior of fluid inclusions in quartz at one atmosphere confining pressure. $J$. Metamorph. Petrol., 7:229-242.
Bodnar R.J., Vityk M.O. 1994. Interpretation of Microthermometric data for $\mathrm{H}_{2} \mathrm{O}-\mathrm{NaCl}$ fluid inclusions. In: De Vivo B., Frezzotti M.L. eds. 1994. Fluid Inclusions in Minerals: Methods and Applications. EUA, Virginia, Blacksburg: Virginia Tech, p. 117-130.

Boiron M.C., Essarraj S., Sellier E., Cathelineau M., Lespinasse M., Poty B. 1992. Identification of fluid inclusions in relation to their host microstructural domains in quartz by cathodoluminescence. Geochim. Cosmochim. Acta, 56:115-185.

Bourdet J., Pironon J., Levresse G., Tritlla J. 2008. Petroleum type determination through homogenization temperature and vapour volume fraction measurements in fluid inclusions. Geofluids, 8:46-59.

Brewster D. 1823. On the existence of two new fluids in the cavities of minerals, which are immiscible, and possess remarkable physical properties. Trans. R. Soc. Edinburgh, 10:1-14.

Burnham C.W. 1997. Chapter 3: Magmas and hydrothermal fluids. In: Barnes H.L. ed. 1997. Geochemistry of Hydrothermal Ore Deposits. 3a Ed. EUA, New York: John Wiley \& Sons Inc., p. 63-358.

Burruss R.C. 1981. Hydrocarbon fluid inclusions in studies of sedimentary diagenesis. In: Hollsster L.S., Crawford M.L. orgs. 1981. Short Course in Fluid Inclusions: Applications to Petrography, 6:138-156.

Burruss R.C. 2003. Chapter 6: Petroleum Fluid Inclusions: An Introduction. In: Samson I., Anderson A., Marshall D. eds. 2003. Fluid Inclusions: Analysis and Interpretation. Canadá, Ottawa: Mineral. Assoc. of Canada, p. 159-174. (Short Course Series, 32.)

Campbell A.R., Hackbart C.J., Plumlee G.S. Petersen, U. 1984. Internal features of ore minerals seen with the infrared microscope. Econ. Geol., 79:1387-1392.

Chen Y., Ge Y., Zhou Z., Zhou Y. 2015. Water, is it necessary for fluid inclusions forming in calcite? J. Pet. Sci. Eng., 133:103-107.

Chi G., Ni P. 2007. Equations for calculation of Na$\mathrm{Cl} /\left(\mathrm{NaCl}+\mathrm{CaCl}_{2}\right)$ ratios and salinities from hydrohalite-melting and ice-melting temperatures in $\mathrm{H}_{2} \mathrm{O}-\mathrm{NaCl}-\mathrm{CaCl}_{2}$ system. Acta Petrol. Sinica, 23:33-37.

Collins, A.G. 1975. Geochemistry of Oilfield Waters. 1a Ed. Amsterdam: Elsevier Scientific Publishing Company, 495 p. (Developments in Petroleum Science, 1.)

CSIRO (Commonwealth Scientic Industrial Research Organisation). 1996. Peter J. Eadington, Mark Lisk, Francis W. Krieger. Identifying oil well sites. US PATENT n 5543616. 6 agosto 1996.

Davy H. 1822. On the state of water and aëriform matter in cavities found in certain crystals. Philos. Trans. R. Soc. London, 112:367-376. 
Diamond L.W. 2003a. Chapter 3: Systematics of $\mathrm{H}_{2} \mathrm{O}$ inclusions. In: Samson I., Anderson A., Marshall D. eds. 2003. Fluid Inclusions: Analysis and Interpretation. Canadá, Ottawa: Mineral. Assoc. of Canada, p. 55-79. (Short Course Series, 32.)

Diamond L.W. 2003b. Chapter 5: Introduction to gas-bearing, aqueous fluid inclusions. In: Samson I., Anderson A., Marshall D. eds. 2003. Fluid Inclusions: Analysis and Interpretation. Canadá, Ottawa: Mineral. Assoc. of Canada, p. 101-158. (Short Course Series, 32.)

Diamond L.W. 2003c. Glossary: Terms and symbols used in fluid inclusion studies. In: Samson I., Anderson A., Marshall D. eds. 2003. Fluid Inclusions: Analysis and Interpretation. Canadá, Ottawa: Mineral. Assoc. of Canada, p. 363-372. (Short Course Series, 32.)

Doppler G., Bakker R.J., Baumgartner M. 2013. Fluid inclusion modification by $\mathrm{H}_{2} \mathrm{O}$ and $\mathrm{D}_{2} \mathrm{O}$ diffusion: the influence of inclusion depth, size, and shape in re-equilibration experiments. Contrib. Mineral. Petrol., 165:1259-1274. 2013.

Eadington P.J., Kempton R.H. 2008. New developments to investigate models of oil accumulation and fluid interactions in reservoirs using data from fluid inclusions. The APPEA J., 48. 1 CD-ROM.

Faiziev A.R., Alidovov B.A. 1976. Thermometry of minerals with perfect cleavage. Soviet Geol. Geophys., 17:118-121.

Frezzotti M.L., Tecce F., Casagli A. 2012. Raman spectroscopy for fluid inclusion analysis. J. Geochem. Exp., 112:1-20.

Gagnon J., Samson I., Fryer B. 2003. Capítulo 12: Laser Ablation ICP-MS Analysis of Fluid Inclusions. In: Samson I., Anderson A., Marshall D. eds. 2003. Fluid Inclusions: Analysis and Interpretation. Canadá, Ottawa: Mineral. Assoc. of Canada, p. 291-319. (Short Course Series, 32.)

George S.C., Lisk M., Eadington P.J. 2004. Fluid inclusion evidence for an early, marine-sourced oil charge prior to gas-condensate migration, Bayu-1, Timor Sea, Australia. Mar. Pet. Geol., 21:1107-1128.

George S.C., Volk H., Ahmed M. 2007. Geochemical analysis techniques and geological applications of oil-bearing fluid inclusions, with some Australian case studies. J. Pet. Sci. Eng., 57:119-138.

Gleeson S.A. 2003. Chapter 9: Bulk analysis of electrolytes in fluid inclusions. In: Samson I., Anderson A., Marshall D. eds. 2003. Fluid Inclusions: Analysis and Interpretation. Canadá, Ottawa: Mineral. Assoc. of Canada, p. 233-247. (Short Course Series, 32.)

Goldstein R.H. 2001. Fluid inclusions in sedimentary and diagenetic systems. Lithos, 55:159-193.

Goldstein R.H. 2003. Capítulo 2: Petrographic analy- sis of fluid inclusions. In: Samson I., Anderson A., Marshall D. eds. 2003. Fluid Inclusions: Analysis and Interpretation. Canadá, Ottawa: Mineral. Assoc. of Canada, p. 9-53. (Short Course Series, 32.)

Goldstein R.H., ReynoldS T.J. 1994. Systematics offluid inclusions in diagenetic minerals. EUA, Tulsa: SEPM, 199 p. (SEPM Short Courses, 31.)

Goldstein R.H. 2001. Fluid inclusions in sedimentary and diagenetic systems. Lithos, 55:159-193.

Hall D.L., Sterner S.M., Bodnar R.J. 1988. Freezing point depression of $\mathrm{NaCl}-\mathrm{KCl}-\mathrm{H}_{2} \mathrm{O}$ solutions. Econ. Geol., 83:197-202.

Hall D.L., Bodnar R.J. 1990. Methane in fluid inclusions from granulites: A product of hydrogen diffusion? Geochim. Cosmochim. Acta, 54:641-651.

Hall D.L., Bodnar R.J., Craig J.R. 1991. Evidence for post entrapment diffusion of hydrogen into peak metamorphic fluid inclusions from the massive sulfide deposits at Ducktown, Tennessee. Am. Mineral., 76:1344-1355.

Hall D.L., Sterner S.M. 1995. Experimental diffusion of hydrogen into synthetic fluid inclusions in quartz. J. Metamorph. Geol., 13:345-355.

Hall D.L., Sterner S.M., Wheeler J.R. 1993. One-atmosphere decrepitation behavior of synthetic fluid inclusions in natural calcite: Implications for the preservation of calcite-hosted inclusions during burial. Arch. Mineral., 49:91-92.

Hanor J.S., Mcintosh J.C. 2007. Diverse origins and timing of formation of basinal brines in the Gulf of Mexico sedimentary basin. Geofluids, 7:227237.

Haynes F.M., Kesler S.E. 1987. Chemical evolution of brines during Mississippi valley-type mineralization: evidence from East Tennessee and Pine Point. Econ. Geol., 82(1):53-71.

Jones D.M., Macleod G. 2000. Molecular Analysis of Petroleum in Fluid Inclusions: A Practical Methodology. Org. Geochem., 31:1163-1173.

Jorge S., Coelho C.E.S., Aquino-Neto F.R. 2011. Analysis of Volatiles in Fluid Inclusions by Direct online Crushing Mass Spectrometry. J. Braz. Chem. Soc., 22(3):437-445.

Karlsen D.A., Nedkvitne T., Larter S.R., Bjørlykke K. 1993. Hydrocarbon composition of authigenic inclusions: applications to elucidation of petroleum reservoir filling history. Geochim. Cosmochim. Acta, 57:3641-3659.

Kurosawa M., Shimano S., Ishii S., Shima K., Kato T. Quantitative trace element analysis of single fluid inclusion by proton-induced X-ray emission (PIXE): Application to fluid inclusions in hydrothermal quartz. Geochim. Cosmochim. Acta, 67:4337-4352.

Liu K., Eadington P.J., Kennard J.M., Middleton H.A., George S.C., Ahmed M., Cope P. 2004. Oil migration in the Vulcan Sub-basin, Timor Sea, in- 
vestigated using GOI and FIS Data. In: Ellis G.K., Baillie P.W., Munson T.J. orgs. Timor Sea Petroleum Geoscience, Proceedings Of The Timor Sea Symposium, Darwin, Northern Territory, 19-20 June 2003. Northern Territory Geological Survey, Special Publication, 1:333-351.

Mavrogenes J.A., Bodnar R.J. 1994. Hydrogen movement into and out of fluid inclusions in quartz: Experimental evidence and geologic implications. Geochim. Cosmochim. Acta, 58:141-148.

Maxwell J.C. 1874. Van der Waals on the Continuity of Gaseous and Liquid States. Nature, 10(259):477480.

McCain W. JR. 1990. The Properties of Petroleum Fluids. 2a Ed. EUA, Oklahoma, Tulsa: PennWell Books Co., 548 p.

McMilans R.K. 1987. The application of fluid inclusions to migration of oil and diagenesis in petroleum reservoirs. App. Geochem., 2:585-603.

Montel F. 1993. Phase equilibria needs for petroleum exploration and production industry. Fluid Phase Equilib., 84:343-367.

Munz I.A. 2001. Petroleum inclusions in sedimentary basins: systematic, analytical methods and applications. Lithos, 55:195-212.

Munz I.A., Yardley B.W., Gleeson S.A. 2002. Petroleum infiltration of high-grade basement, South Norway: Pressure-Temperature-time-composition (P-T-t-X) constraints. Geofluids, 2:41-53.

Murray R.C. 1957. Hydrocarbon fluid inclusions in quartz. AAPG Bull., 41:950-956.

Naden J. 1996. CalcicBrine: a Microsoft Excel 5.0 add-in for calculating salinities from microthermometric data in the system $\mathrm{NaCl}-\mathrm{CaCl}_{2}-\mathrm{H}_{2} \mathrm{O}$. In: PACROFI, 6, Abstracts... EUA, Madison: University of Wisconsin-Madison, p. 97-98.

Narr W., Burruss R.C. 1984. Origin of reservoir fractures in Little Knife Field, North Dakota. AAPG Bull., 68:1087-1100.

Naumov V.B., Balitskiy V.S., Khetchikov L.N. 1966. Correlation of the temperatures of formation, homogenization and decrepitation of gas-fluid inclusions. Dokl. Akad. Nauk SSSR, 171:146-148.

Oakes C.S., Bodnar R.J., Simonson J.M. 1990. The system $\mathrm{NaCl}-\mathrm{CaCl}_{2}-\mathrm{H}_{2} \mathrm{O}: 1$. The ice liquidus at $1 \mathrm{~atm}$ total pressure. Geochim. Cosmochim. Acta, 54:603-610.

Pang L.S.K., George S., Quezada R.A. 1998. A study of the gross compositions of oil-bearing fluid inclusions using high performance liquid chromatography. Org. Geochem., 29(5-7):1149-1161.

Peng D.Y., Robinson D.B. 1976. A new two-constant equation of state. Ind. Eng. Chem. Fundam., 15:59-64.

Pestilho A.L.S. 2008. Depósito de Cu-(Zn-Ni) do Alvo Castanha, Província Mineral de Carajás, PA: Evolução Paragenética e dos Fluidos Mineralizantes.
Campinas, Univ. Est. Campinas, Inst. Geoc. 85p. (Trab. Concl. Curso).

Ping H., Thiéry R., Chen H. 2011. Thermodynamic modeling of petroleum inclusions: the prediction of the saturation pressure of crude oils. Geofluids, 11:328-340.

Pironon J., Thiéry R., Ayt Ougougdal M., Teinturier S., Beaudoin G., Walgenwitz F. 2001. FT-IR measurements of petroleum fluid inclusions: methane, $\mathrm{n}$-alkanes and carbon dioxide quantitative analysis. Geofluids, 1:2-10.

Prezbindowski D.R., Larese R.E. 1987. Experimental stretching of fluid inclusions in calcite - Implications for diagenetic studies. Geology, 15:333-336.

Prezbindowski D.R., Tapp J.B. 1991. Dynamics of fluid inclusion alteration in sedimentary rocks: a review and discussion. Org. Geochem., 17:131-142.

Robert F., Kelly W.C. 1987. Ore-forming fluids in Archean gold-bearing quartz veins at the Sigma Mine, Abitibi greenstone belt, Quebec, Canada. Econ. Geol., 82:1464-1482.

Roedder E. 1958. Technique for the extraction and partial chemical analysis of fluid-filled inclusions from minerals. Econ. Geol., 53(3):235-267.

Roedder E. 1962. Studies of fluid inclusions I: Low temperature application of a dual-purpose freezing and heating stage. Econ. Geol., 57:1054-1061.

Roedder E. 1963a. Studies of fluid inclusions II: Freezing data and their interpretation. Econ. Geol, 58(2):167-208.

Roedder E. 1963b. Studies of fluid inclusions III: Extraction and quantitative analysis of inclusions in the milligram range. Econ. Geol., 58(3):353-374.

Roedder E. 1965. Evidence from fluid inclusions as to the nature of the ore-forming fluids. In: Symposium On Problems Of Postmagmatic Ore Deposition. Proceedings... Prague, Serviço Geológico da Czechoslovakia, 2:375-384. il. 13 pranchas.

Roedder E. 1971. Fluid inclusion studies on the porphyry-type ore deposits at Bingham, Utah, Butte, Montana, and Climax, Colorado. Econ. Geol., 66:98-120.

Roedder E. 1984. Fluid inclusions. EUA, Washington, D.C.: Mineral. Soc. of Am., 644 p. (Reviews in Mineralogy, 12.)

Roedder E., Bodnar R.J. 1980. Geologic pressure determinations from fluid inclusion studies. Annu. Rev. Earth Planet. Sci., 8:263-301.

Roedder E., Bodnar R.J. 1997. Capítulo 13: Fluid inclusion studies of hydrothermal ore deposits. In: Barnes H.L. ed. 1997. Geochemistry of Hydrothermal Ore Deposits. 3a Ed. EUA, New York: John Wiley \& Sons Inc., p. 657-697.

Roedder E., Skinner B.J. 1968. Experimental evidence that fluid inclusions do not leak. Econ. Geol., 63(7):715-729.

Sabouraud C. 1994. Capítulo 5: Solid and fluid in- 
clusions of evaporites; gypsum. In: Majithia M. ed. 1994. Evaporite sequences in petroleum exploration. Tradução de India Kohli. França, Paris: Éditions Technip, p. 136-145. (Geological Methods, 1.)

Salvi S., Williams-Jones A.E. 2003. Capítulo 10: Bulk analysis of volatiles in fluid inclusions. In: Samson I., Anderson A., Marshall D. eds. 2003. Fluid Inclusions: Analysis and Interpretation. Canadá, Ottawa: Mineral. Assoc. of Canada, p. 247-278. (Short Course Series, 32.)

Samson I., Samson I., Anderson A., Marshall D. eds. 2003. Fluid Inclusions: Analysis and Interpretation. Canadá, Ottawa: Mineral. Assoc. of Canada, 374 p. (Short Course Series, 32.)

Schiffries C.M. 1990. Liquid-absent, aqueous fluid inclusions and phase equilibria in the system $\mathrm{CaCl}_{2}-\mathrm{NaCl}-\mathrm{H}_{2} \mathrm{O}$. Geochim. Cosmochim. Acta, 54:611-619.

Soave G. 1972. Equilibrium constants from a modified Redlich-Kwong equation of state. Chem. Eng. Sci., 27:1197-1203.

Sorby H.C. 1858. On the microscopic structure of crystals, indicating the origin of minerals and rocks. Geol. Soc. London Quart. J., 14(1):453-500.

Stasiuk L.D., Snowdon L.R. 1997. Fluorescence micro-spectrometry of synthetic and natural hydrocarbon fluid inclusions: crude oil chemistry, density and application to petroleum migration. Org. Geochem., 12:229-241.

Steele-Macinnis M., Bodnar R.J. 2013. Effect of the vapor phase on the salinity of halite-bearing aqueous fluid inclusions estimated from the halite dissolution temperature. Geochim. Cosmochim. Acta, 115:205-216.

Steele-Macinnis M., Bodnar R.J., Naden J. 2011. Numerical model to determine the composition of $\mathrm{H}_{2} \mathrm{O}-\mathrm{NaCl}-\mathrm{CaCl}_{2}$ fluid inclusions based on microthermometric and microanalytical data. Geochim. Cosmochim. Acta, 75:21-40.

Sterner S.M., Hall D.L., Bodnar R.J. 1988. Synthetic fluid inclusions. V. Solubility relations in the system $\mathrm{NaCl}-\mathrm{KCl}-\mathrm{H}_{2} \mathrm{O}$ under vapor-saturated conditions. Geochim. Cosmochim. Acta, 52:989-1005.

Sterner S.M., Chou I-M., Downs R.T., Pitzer K.S. 1992. Phase relations in the system NaCl-KCl-H2O: V. Thermodynamic-PTX analysis of solid-liquid equilibria at high temperatures and pressures. Geochim. Cosmochim. Acta, 56:2295-2309.

Stoffell B., Appold M.S., Wilkinson J.J., Mcclean N.A., Jeffries T.E. 2008. Geochemistry and evolution of Mississippi valley-type mineralizing brines from the Tri-State and Northern Arkansas districts determined by LA-ICP-MS microanalysis of fluid inclusions. Econ. Geol., 103:1411-1435.

Suchý V., Dobeš P., Sýkorová I., Machovič V., Stejskal M., Kroufek J., Chudoba J., Matロjovský L., Havelcová M., Matysová P. 2010. Oil-bearing inclusions in vein quartz and calcite and, bitumens in veins: Testament to multiple phases of hydrocarbon migration in the Barrandian basin (lower Palaeozoic), Czech Republic. Mar. Pet. Geol., 27:285-297.

Teinturier S., Elie M., Pironon J. 2003. Oil-cracking processes evidence from synthetic petroleum inclusions. J. Geoch. Explor., 78-79:421-425.

Teinturier S., Pironon J. 2004. Experimental growth of quartz in petroleum environment. Part I: Procedures and Fluid Trapping. Geochim. Cosmochim. Acta, 68(11):2495-2507.

Thiéry R. 2011. Publicação eletrônica [mensagem pessoal]. Mensagem recebida por < andrepestilho@ gmail.com > em 28 de novembro de 2011.

Thiéry R., Pironon J., Walgenwitz F., Montel F. 2000. PIT (Petroleum Inclusion Thermodynamic): a new modeling tool for the characterization of hydrocarbon fluid inclusions from volumetric and microthermometric measurements. J. Geochem. Exp., 69-70:701-704.

Thiéry R., Pironon, J., Walgenwitz, F., Montel F. 2002. Individual characterization of petroleum fluid inclusions (composition and P-T trapping conditions) by microthermometry and confocal laser scanning microscopy: inferences from applied thermodynamics of oils. Mar. Pet. Geol., 19:847-59.

Tissot B.P., Welte D.H. 1984. Petroleum Formation and Occurrence: A New Approach to Oil and Gas Exploration. 2a Ed. Alemanha, Berlin: Springer-Verlag. 699 p.

Tugarinov A.I., Naumov V.B. 1970. Dependence of the decrepitation temperature of minerals on the composition of their gas-liquid inclusions and hardness. Dokl. Akad. Nauk SSSR, 195: 112-114.

Ulrich M.R., Bodnar R.J. 1988. Systematics of stretching fluid inclusions. II. Barite at one atmosphere confining pressure. Econ. Geol., 83:1037-1046.

Van Den Kerkhof A.M., Hein U.F. 2001. Fluid inclusion petrography. Lithos, 55:27-47.

Vanko D.A. 1988. Temperature, pressure, and composition of hydrothermal fluids, with their bearing on the magnitude of tectonic uplift at mid-ocean ridges, inferred from fluid inclusions in oceanic layer 3 rocks. J. Geophys. Res., 93:4595-4611.

Vanko D.A., Bodnar R.J., Sterner S.M. 1988. Synthetic fluid inclusions: VIII. Vapor-saturated halite solubility in part of the system $\mathrm{NaCl}-\mathrm{CaCl}_{2}-\mathrm{H}_{2} \mathrm{O}$, with application to fluid inclusions from oceanic hydrothermal systems. Geochim. Cosmochim. Acta, 52:2451-2456.

VTFLinc. 1997. User's guide. Dinamarca, Lingby: CALSEP A/S, Chemical Engineering Consulting.

Viti C., Frezzotti M-L. 2001. Transmission electron microscopy applied to fluid inclusion investigations. Lithos, 55:125-138. 
Vityk M.O., Bodnar R.J., Doukhan J-C. 2000. Synthetic fluid inclusions. XV. TEM investigation of plastic fow associated with reequilibration of fluid inclusions in natural quartz. Cont. Min. Petrol., 139:285-297.

Volk H., Fuentes D., Fuerbach A., Miese C., Koehler W., Bärsch N., Barcikowski S. 2010. First on-line analysis of petroleum from single inclusion using ultrafast laser ablation. Org. Geochem., 41:74-77.

Wang Z., Yang C., Fingas M., Hollebone B., Yim U.H., Oh J.R. 2007. 3 Petroleum Biomarker Fingerprinting for Oil Spill Characterization and Source Identification. In: Wang Z., Stout S.A. eds.
2007. Oil Spill Environmental Forensics: Fingerprinting And Source Identification. EUA, New York: Elsevier/Academic Press, p. 73-146.

Wilkinson J.J. 2001. Fluid inclusions in hydrothermal ore deposits. Lithos, 55:229-272.

Williams-Jones A.E., Samson I.M. 1990. Theoretical estimation of halite solubility in the system $\mathrm{Na}$ $\mathrm{Cl}-\mathrm{CaCl}_{2}-\mathrm{H}_{2} \mathrm{O}$ : applications to fluid inclusions. Can. Mineral., 28:299-304.

$\mathrm{Xu}, \mathrm{G} .2000$. Fluid inclusions with $\mathrm{NaCl}-\mathrm{CaCl}_{2}-\mathrm{H}_{2} \mathrm{O}$ composition from the Cloncurry hydrothermal system, NW Queensland, Australia. Lithos, 53:21-35.

Resumo: Este trabalho visa introduzir as bases conceituais, a filosofia e o estado da arte atual do estudo de inclusões fluidas. 0 texto inicia com uma breve visão geral de desenvolvimento histórico e do estado atual desse campo de estudo. A partir deste ponto são abordados os principais conceitos quanto a origem das inclusões fluidas e as premissas básicas para interpretação dos parâmetros derivados das inclusões (Regras de Roedder). Por fim, são apresentadas as bases da intepretação dos dados microtermométricos, com exemplos de sistemas químicos de inclusões fluidas aquosas, além de uma revisão dos conceitos que envolvem o estudo das inclusões fluidas de petróleo.

PALAVRAS-CHAVE: Geologia, inclusões fluidas, geologia econômica, geologia do petróleo. 